\title{
Collective modes in anisotropic systems
}

\author{
Margaret E. Carrington $\odot,{ }^{1,2}$ Bailey M. Forster ${ }^{1}$ and Sofiya Makar ${ }^{3}$ \\ ${ }^{1}$ Department of Physics and Astronomy, Brandon University, Brandon, Manitoba R7A 6A9, Canada \\ ${ }^{2}$ Winnipeg Institute for Theoretical Physics, Winnipeg, Manitoba, Canada \\ ${ }^{3}$ Department of Mathematics, Brandon University, Brandon, Manitoba R7A 6A9, Canada
}

(Received 23 July 2021; accepted 29 September 2021; published 22 December 2021)

\begin{abstract}
We study collective modes in anisotropic plasmas of quarks and gluons using a quasiparticle picture and a hard-loop approximation. We use a general class of anisotropic distribution functions, and we consider chirally asymmetric systems. We introduce a complete tensor basis to decompose the gluon polarization tensor into a set of nine scalar functions. We derive and solve the corresponding dispersion equations. Imaginary modes are particularly important because of their potential influence on plasma dynamics. We explore in detail their dependence on the chiral chemical potential and the parameters that characterize the anisotropy of the system. We show that our generalized distributions produce dispersion relations that are much richer in structure than those obtained with a simple one-parameter deformation of an isotropic distribution. In addition, the size and domain of the imaginary solutions are enhanced, relative to those obtained with a one-parameter deformation. Finally, we show that the influence of even a very small chiral chemical potential is significantly magnified when anisotropy is present.
\end{abstract}

DOI: 10.1103/PhysRevC.104.064908

\section{INTRODUCTION}

We consider a relativistic plasma of charged particles that is not necessarily in equilibrium, but is in the regime where a quasiparticle description is valid. We use an effective phasespace description in terms of distribution functions that are not assumed thermal or isotropic. A convenient parametrization for the distribution function of a spheroidally anisotropic system was introduced in [1,2]. The idea is to introduce one parameter that effectively stretches or squeezes the spherically symmetric isotropic distribution in one direction. This parametrization is interesting in the context of heavy-ion collisions where one expects the parton distribution that is produced by the collision to be squeezed along the beam axis. Dispersion relations are obtained from the poles of the dressed propagators of the partonic quasiparticles. One finds that the spectrum of collective excitations is much richer than the spectrum of a system in thermal equilibrium. It is especially interesting that there are imaginary modes, which are associated with instabilities and could play an important role in the thermalization of the plasma [3-8]. Anisotropic plasmas have been studied intensively in the context of quark-gluon plasmas, which thermalize earlier than expected, for reasons that are as yet unclear. Collective modes have been calculated for both gluons [1,2,9] and quarks [10]. An ellipsoidal gener-

Published by the American Physical Society under the terms of the Creative Commons Attribution 4.0 International license. Further distribution of this work must maintain attribution to the author(s) and the published article's title, journal citation, and DOI. Funded by $S C O A P^{3}$. alization was developed in [11,12]. These distributions have been used to study various properties of quark-gluon plasma (QGP) including transport coefficients [13-16], heavy-quark bound states [17-20], and photon and dilepton production [10,21-24].

Isotropic plasmas also support imaginary collective modes, if there is a chiral imbalance [25]. Chiral plasmas with spheroidal anisotropy were studied in Ref. [26]. Chiral systems are of interest in a wide variety of different situations in particle physics, nuclear physics, condensed matter physics, and cosmology. One important example is the chiral magnetic effect (CME), which is the production of a parity-violating current in a plasma, in the presence of a magnetic field and an asymmetry between left- and right-handed fermions [27]. A chirally asymmetric plasma can be described using a chiral chemical potential defined as $\mu_{5} \equiv\left(\mu_{R}-\mu_{L}\right) / 2$, where $\mu_{L}$ and $\mu_{R}$ are the chemical potentials of the left- and righthanded fermions. The CME is particularly interesting in the context of heavy-ion collisions because it has been argued that the quark gluon plasma that is produced in noncentral collisions may contain regions where $\mu_{5}$ is locally finite [28-32]. Instabilities in chiral plasmas have also been studied in electroweak theory at large lepton chemical potential [33] and in the context of the early universe at $T \gg \mu_{5}$ [34].

Collective modes can be studied using either kinetic theory or effective field theories. The equivalence of the kinetic theory method and a hard thermal loop (HTL) effective theory approach, for isotropic and chirally symmetric systems, was shown in [35-37]. For anisotropic chirally symmetric systems, the connection between kinetic theory and a generalization of the HTL effective theory, which is called a hard-loop (HL) effective theory, has also been established [38,39]. Isotropic chiral kinetic theories have been developed 
in [40-44], and applied to a variety of physical problems; see for example [45-47].

In this paper we use anisotropic distributions that are more general than those used in previous works, and we study the spectrum of collective modes in anisotropic plasmas with nonzero chiral chemical potential. The method can be applied generally to either a QED plasma of ultrarelativistic electrons and positrons or a QGP. We use natural units where $\hbar=c=1$. The indices $i, j, k=1,2,3$ and $\mu, v=0,1,2,3$ label, respectively, the Cartesian spatial coordinates and those of Minkowski space. Our metric is mostly minus $g_{\mu \nu}=$ $(1,-1,-1,-1)_{\text {diag. }}$. We use capital letters for four-vectors so that, for example, $P^{2}=p_{0}^{2}-\vec{p} \cdot \vec{p}=p_{0}^{2}-p^{2}$. We define the unit vector $\hat{p}=\vec{p} /|\vec{p}|$ and we will also use $\hat{p}_{0}=p_{0} / p$. Real solutions to dispersion equations will be denoted $\omega(\vec{p})$ and imaginary solutions are written $i \gamma(\vec{p})$.

\section{ISOTROPIC FORMALISM}

In vacuum the photon polarization tensor can be written in terms of one scalar function using a transverse projection operator as $\Pi^{\mu \nu}\left(p_{0}, \vec{p}\right)=\left(P^{2} g^{\mu \nu}-P^{\mu} P^{v}\right) \Pi\left(p_{0}, \vec{p}\right)$. At finite temperature the rest frame of the heat bath, which we define with the four-vector $n^{\mu}=(1,0,0,0)$, breaks Lorentz invariance. Timelike axial gauge (TAG) is particularly useful at finite temperature, because the gauge condition is imposed in the heat bath rest frame. In TAG we only have to consider the spatial components of the propagator, for which the Dyson equation has the form

$$
\begin{aligned}
D^{i j-1}\left(p_{0}, \vec{p}\right) & =D_{0}^{i j-1}\left(p_{0}, \vec{p}\right)-\Pi^{i j}\left(p_{0}, \vec{p}\right) \\
& =P^{2} \delta^{i j}+p^{2} \hat{p}^{i} \hat{p}^{j}-\Pi^{i j}\left(p_{0}, \vec{p}\right) .
\end{aligned}
$$

In an equilibrated chirally symmetric plasma, the polarization tensor is symmetric and has two independent components, one transverse and the other longitudinal with respect to the three-vector $\vec{p}$. An isotropic system with finite chiral chemical potential was first considered in [25], and we review the formalism presented in that paper below. In the presence of finite $\mu_{5}$ the polarization tensor has an additional asymmetric component and can be written

$$
\begin{aligned}
\Pi^{i j}\left(p_{0}, \vec{p}\right)= & \left(\delta^{i j}-\hat{p}^{i} \hat{p}^{j}\right) \Pi_{T}\left(p_{0}, \vec{p}\right) \\
& +\hat{p}^{i} \hat{p}^{j} \Pi_{L}\left(p_{0}, \vec{p}\right)+i \epsilon^{i j m} \hat{p}^{m} \Pi_{A}\left(p_{0}, \vec{p}\right) .
\end{aligned}
$$

The propagator, obtained from inverting the Dyson equation, is

$$
\begin{aligned}
D^{i j}\left(p_{0}, \vec{p}\right)= & \left(\delta^{i j}-\hat{p}^{i} \hat{p}^{j}\right) \frac{\left[P^{2}-\Pi_{T}\left(p_{0}, \vec{p}\right)\right]}{\left[P^{2}-\Pi_{T}\left(p_{0}, \vec{p}\right)\right]^{2}-\Pi_{A}^{2}\left(p_{0}, \vec{p}\right)} \\
& +\hat{p}^{i} \hat{p}^{j} \frac{1}{p_{0}^{2}-\Pi_{L}\left(p_{0}, \vec{p}\right)} \\
& +i \epsilon^{i j m} \hat{p}^{m} \frac{\Pi_{A}\left(p_{0}, \vec{p}\right)}{\left[P^{2}-\Pi_{T}\left(p_{0}, \vec{p}\right)\right]^{2}-\Pi_{A}^{2}\left(p_{0}, \vec{p}\right)} .
\end{aligned}
$$

The dispersion relations are obtained from the dispersion equations which give the poles of the retarded propagator.
From Eq. (3) we have that the dispersion equations for an isotropic chiral plasma are

$$
\begin{aligned}
p_{0}^{2}-\Pi_{L}\left(p_{0}, \vec{p}\right) & =0, \\
P^{2}-\left[\Pi_{T}\left(p_{0}, \vec{p}\right)+\Pi_{A}\left(p_{0}, \vec{p}\right)\right] & =0, \\
P^{2}-\left[\Pi_{T}\left(p_{0}, \vec{p}\right)-\Pi_{A}\left(p_{0}, \vec{p}\right)\right] & =0 .
\end{aligned}
$$

Throughout this paper we refer always to the retarded polarization tensor, and we will not include any subscripts to indicate this.

Our notation for the equilibrium distribution function is [see Eq. (A4)]

$$
\begin{aligned}
& n(k)=n^{+}(k)=\frac{1}{e^{\beta(k-\mu)}+1}, \\
& \bar{n}(k)=n^{-}(-k)=\frac{1}{e^{\beta(k+\mu)}+1} .
\end{aligned}
$$

The distributions for right/left-handed particles/antiparticles are written $n_{R}(k), \bar{n}_{R}(k), n_{L}(k)$, and $\bar{n}_{L}(k)$, where, for example, $n_{R}(k)=\left.n(k)\right|_{\mu=\mu_{R}}$.

We calculate the 1-loop photon polarization tensor in the HTL approximation (see Appendix A for details). The contribution from right-handed fermions is

$$
\begin{aligned}
\Pi_{R}^{i j}\left(p_{0}, \vec{p}\right)= & \Pi_{R \text { even }}^{i j}\left(p_{0}, \vec{p}\right)+\Pi_{R \text { odd }}^{i j}\left(p_{0}, \vec{p}\right) \\
\Pi_{R \text { even }}^{i j}\left(p_{0}, \vec{p}\right)= & 2 g^{2} \int \frac{d^{3} k}{(2 \pi)^{3}} \frac{n_{R}(k)+\bar{n}_{R}(k)}{k} \\
& \times\left(\delta^{i j}+\frac{v^{i} p^{j}+p^{i} v^{j}}{P \cdot V+i \epsilon}-\frac{P^{2} v^{i} v^{j}}{(P \cdot V+i \epsilon)^{2}}\right), \\
\Pi_{R \text { odd }}^{i j}\left(p_{0}, \vec{p}\right)= & i g^{2} P^{2} \epsilon^{i j m} \int \frac{d^{3} k}{(2 \pi)^{3}} \\
& \times \frac{n_{R}(k)-\bar{n}_{R}(k)}{k^{2}} \frac{\left(p_{0} v^{m}-p^{m}\right)}{(P \cdot V+i \epsilon)^{2}}
\end{aligned}
$$

and the corresponding expression for left-handed fermions is obtained from the transformation $\mu_{R} \rightarrow \mu_{L}$. We use $P \cdot V=$ $p_{0}-\vec{p} \cdot \vec{v}$ and $\vec{k} / \sqrt{k^{2}+m^{2}} \approx \hat{k} \equiv \vec{v}$ since massless fermions are consistent with the hard-loop approximation. It is straightforward to modify the HTL integrals to the case of a QCD plasma by changing the QED coupling constant to the QCD one, and using [48]

$$
\begin{aligned}
& n(k)+\bar{n}(k) \rightarrow \frac{N_{f}}{2}\left[n_{q}(k)+\bar{n}_{q}(k)\right]+N_{c} n_{g}(k), \\
& n(k)-\bar{n}(k) \rightarrow \frac{N_{f}}{2}\left[n_{q}(k)-\bar{n}_{q}(k)\right],
\end{aligned}
$$

where the subscripts $q$ and $g$ refer to quark (or antiquark) and gluon distribution functions. Equations (8) and (9) can be rewritten in a form that is sometimes more useful by integrating by parts. A straightforward calculation gives

$$
\begin{aligned}
\Pi_{R \text { even }}^{i j}\left(p_{0}, \vec{p}\right)= & -2 g^{2} \int \frac{d^{3} k}{(2 \pi)^{3}} \frac{\partial\left[n_{R}(k)+\bar{n}_{R}(k)\right]}{\partial k^{m}} \\
& \times v^{i}\left(\delta^{j m}+\frac{v^{j} p^{m}}{P \cdot V}\right),
\end{aligned}
$$




$$
\begin{aligned}
\Pi_{R \mathrm{ddd}}^{i j}\left(p_{0}, \vec{p}\right)= & -i g^{2} \epsilon^{i j m} \int \frac{d^{3} k}{(2 \pi)^{3}} \frac{1}{k} \frac{\partial}{\partial k^{l}}\left[n_{R}(k)-\bar{n}_{R}(k)\right] \\
& \times\left(p_{0} \delta^{l m}+\frac{\left(p_{0} v^{m}-p^{m}\right) p^{l}}{P \cdot V}\right)
\end{aligned}
$$

From the definitions in Eqs. (A9) and (A10) one finds

$$
\begin{aligned}
\Pi^{i j}\left(p_{0}, \vec{p}\right)= & \frac{1}{2}\left[\Pi_{R}^{i j}\left(p_{0}, \vec{p}\right)+\Pi_{L}^{i j}\left(p_{0}, \vec{p}\right)\right] \\
= & \frac{1}{2}\left(\Pi_{R \text { even }}^{i j}+\Pi_{L \text { even }}^{i j}\right)+\frac{1}{2}\left(\Pi_{R \text { odd }}^{i j}+\Pi_{L \text { odd }}^{i j}\right) \\
= & \frac{1}{2}\left(\Pi_{R \text { even }}^{i j}+\left.\Pi_{R \text { even }}^{i j}\right|_{\mu_{R} \rightarrow \mu_{L}}\right) \\
& +\frac{1}{2}\left(\Pi_{R \text { odd }}^{i j}+\left.\Pi_{R \text { odd }}^{i j}\right|_{\mu_{R} \rightarrow-\mu_{L}}\right)
\end{aligned}
$$

The functions $\Pi_{T}\left(p_{0}, \vec{p}\right), \Pi_{L}\left(p_{0}, \vec{p}\right)$, and $\Pi_{A}\left(p_{0}, \vec{p}\right)$ in Eqs. (2) and (3) are calculated by applying the projection operators $\mathcal{P}_{T}^{i j}=\left(\delta^{i j}-\hat{p}^{i} \hat{p}^{j}\right) / 2, \quad \mathcal{P}_{L}^{i j}=\hat{p}^{i} \hat{p}^{j}$, and $\mathcal{P}_{A}^{i j}=-i \epsilon^{i j m} \hat{p}^{m} / 2$ to $\Pi^{i j}\left(p_{0}, \vec{p}\right)$. The resulting expressions for the three scalar components of the polarization tensor are

$$
\begin{aligned}
& \Pi_{T}\left(p_{0}, \vec{p}\right)=m_{D}^{2} \frac{p_{0}^{2}}{2 p^{2}}\left[1-\frac{P^{2}}{2 p_{0} p} \ln \left(\frac{p_{0}+p+i \epsilon}{p_{0}-p+i \epsilon}\right)\right], \\
& \Pi_{L}\left(p_{0}, \vec{p}\right)=-m_{D}^{2} \frac{p_{0}^{2}}{p^{2}}\left[1-\frac{p_{0}}{2 p} \ln \left(\frac{p_{0}+p+i \epsilon}{p_{0}-p+i \epsilon}\right)\right], \\
& \Pi_{A}\left(p_{0}, \vec{p}\right)=-g^{2} \frac{\mu_{5} P^{2}}{2 \pi^{2} p}\left[1-\frac{p_{0}}{2 p} \ln \left(\frac{p_{0}+p+i \epsilon}{p_{0}-p+i \epsilon}\right)\right],
\end{aligned}
$$

where we have used $\mu_{5}=\left(\mu_{R}-\mu_{L}\right) / 2$ and defined the Debye mass parameter

$$
\begin{aligned}
m_{D}^{2} & =2 g^{2} \int \frac{d^{3} k}{(2 \pi)^{3}} \frac{1}{k}\left([n(k)+\bar{n}(k)]_{\mu_{R}}+[n(k)+\bar{n}(k)]_{\mu_{L}}\right) \\
& =g^{2}\left(\frac{T^{2}}{3}+\frac{1}{2 \pi^{2}}\left(\mu_{R}^{2}+\mu_{L}^{2}\right)\right) .
\end{aligned}
$$

The transverse and longitudinal components of the polarization tensor are the familiar HTL results, and in the chirally symmetric limit $\mu_{R}=\mu_{L}$ give $\mu_{5}=0$ and therefore $\Pi_{A}\left(p_{0}, \vec{p}\right)=0$. Throughout the rest of this paper we use the shorthand notation $\hat{\mu}_{5}=g^{2} \mu_{5} /\left(2 \pi^{2}\right)$. In all of our numerical calculations we set the Debye mass to one, which is equivalent to defining all dimensionful quantities in units of the Debye mass.

The dispersion relations are obtained by solving Eqs. (4)(6), where the functions $\Pi_{L}\left(p_{0}, \vec{p}\right), \Pi_{T}\left(p_{0}, \vec{p}\right)$, and $\Pi_{A}\left(p_{0}, \vec{p}\right)$ are given in Eq. (14). There are pure real and pure imaginary solutions that appear in positive and negative pairs. Equation (4) has two real solutions which are just the usual longitudinal HTL modes, and are written $\pm \omega_{L}(p)$. Equation (5) also has two real solutions, which we denote $\pm \omega_{+}(p)$. Equation (6) has two real solutions, which we call $\pm \omega_{-}(p)$, and two extra solutions that appear at values of the wave vector below the critical value $p_{\text {crit }}=\hat{\mu}_{5}$. These solutions are pure imaginary and denoted $\pm i \gamma_{-}(p)$. The critical wave vector at which the imaginary solutions appear can be found with a Nyquist analysis (see Appendix B for details). When $\hat{\mu}_{5}=0$ we have $\omega_{+}(p)=\omega_{-}(p)=\omega_{T}(p)$, where $\omega_{T}(p)$ is the transverse HTL
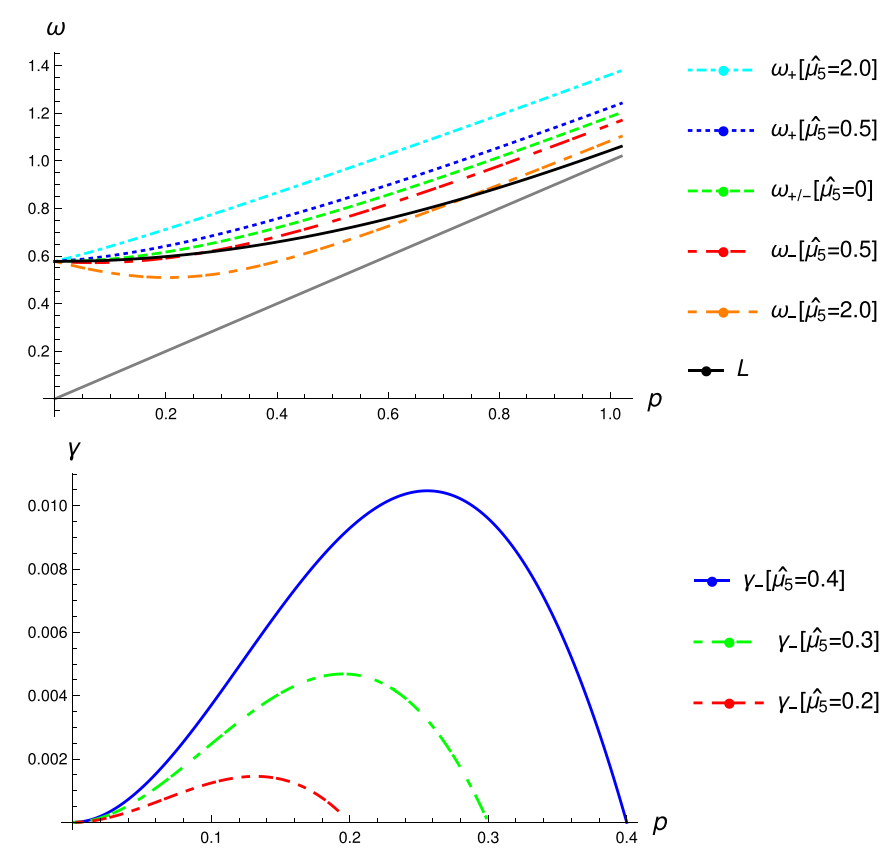

FIG. 1. Real $(\omega)$ and imaginary $(\gamma)$ solutions to the dispersion equations (4)-(6) for different values of $\hat{\mu}_{5}=g^{2} \mu_{5} /\left(2 \pi^{2}\right)$. The units are defined by setting $m_{D}=1$. The diagonal gray line in the top figure is the light cone.

mode. In Fig. 1 we show solutions to Eqs. (4)-(6) for different values of the parameter $\hat{\mu}_{5}$.

\section{ANISOTROPIC FORMALISM}

\section{A. Distribution function}

Next we consider the situation that the momentum distribution is not isotropic. A simple way to account for momentum anisotropy is to replace the distribution function in Eqs. (8) and (9) using $n(k) \rightarrow n(\vec{k})=C_{\xi} n\left(k H_{\xi}(\vec{v})\right)$, where $\vec{v}=\vec{k} / k$, and similarly for $\bar{n}(k)$. The subscript $\xi$ indicates dependence on a set of anisotropy parameters that can be used to construct a distribution that is deformed relative to the isotropic one. The factor $C_{\xi}$ is a normalization and can be defined in different ways depending on the calculation being done. Our choice is explained at the end of this section.

We can construct a completely general expression for the function $H_{\xi}(\vec{v})$ as a sum of terms that are products of anisotropy parameters and dot products of the vector $\vec{v}$ with two perpendicular unit vectors, which we will write $\hat{n}_{3}$ and $\hat{n}_{1}$. We are primarily interested in distributions of partons produced in heavy-ion collisions. In this context, we will take $\hat{n}_{3}$ to define the beam axis and $\hat{n}_{1}$ to give the direction of transverse anisotropy. We restrict to functions that satisfy the condition $H_{\xi}(\vec{v})=H_{\xi}(-\vec{v})$ and use an expression of the form

$$
\begin{aligned}
H_{\xi}^{2}(\vec{v})= & \xi_{0}+\xi_{2}\left(\vec{n}_{1} \cdot \vec{v}\right)^{2}+\xi_{9}\left(\vec{n}_{3} \cdot \vec{v}\right)^{2}+\xi_{6}\left(\vec{n}_{1} \cdot \vec{v}\right)\left(\vec{n}_{3} \cdot \vec{v}\right) \\
& +\xi_{4}\left(\vec{n}_{1} \cdot \vec{v}\right)^{4}+\xi_{8}\left(\vec{n}_{1} \cdot \vec{v}\right)^{3}\left(\vec{n}_{3} \cdot \vec{v}\right) \\
& +\xi_{11}\left(\vec{n}_{1} \cdot \vec{v}\right)^{2}\left(\vec{n}_{3} \cdot \vec{v}\right)^{2}+\xi_{13}\left(\vec{n}_{1} \cdot \vec{v}\right)\left(\vec{n}_{3} \cdot \vec{v}\right)^{3} \\
& +\xi_{14}\left(\vec{n}_{3} \cdot \vec{v}\right)^{4}
\end{aligned}
$$


When $\xi_{0}=1$ and $\xi_{i \neq 0}=0$, we have $H_{\xi}^{2}(\vec{v})=1$, and the distribution is isotropic. For an arbitrary choice of the anisotropy parameters $\xi_{i}$, the isotropic distribution is expanded in the direction of $\vec{v}$ if $H_{\xi}(\vec{v})<1$, and contracted if $H_{\xi}(\vec{v})>1$. The values of the anisotropy parameters must be chosen so that $H_{\xi}^{2}(\vec{v})$ is positive for all orientations of the vector $\vec{v}$, which is equivalent to the requirement that $H_{\xi}(\vec{v})$, and therefore the argument of the distribution function, is real and positive. In Sec. III C we will show that our expression (16) is equivalent to including spherical harmonics up to second order.

Equation (16) includes the parametrizations used in several previous calculations. In the original work of Ref. [1] the authors used $\xi_{0}=1$ and $\xi_{9} \in(-1, \infty)$ with all other parameters set to zero. The chosen value of $\xi_{9}$ allows one to consider either a slightly prolate momentum distribution $\left(-1<\xi_{9}<0\right)$, which is elongated along the beam axis, or an arbitrarily oblate distribution $\left(\xi_{9}>0\right)$, which is squeezed in the direction of the beam axis. In the calculations of Refs. [11,12], where ellipsoidal asymmetry was included for the first time, the authors used $\xi_{0}=1$ and various values of $\xi_{2}$ and $\xi_{9}$ with all other parameters zero. In Ref. [9] the authors considered a one-parameter deformation using $\xi_{0}=1+\sigma$ and $\xi_{9}=-\sigma$ with $\sigma \in(-1, \infty)$. The resulting distribution is slightly oblate for $-1<\sigma<0$ and prolate to any degree for $\sigma>0$.

Our definition (16) can be extended by including terms with higher-order products of the scalar products $\left(\hat{n}_{3} \cdot \vec{v}\right)$ and $\left(\hat{n}_{3} \cdot \vec{v}\right)$. The only restriction we impose, besides positivity, is the condition $H_{\xi}(\vec{v})=H_{\xi}(-\vec{v})$. This means there are no terms where the sum of the exponents is odd, like for example $\left(\hat{n}_{3} \cdot \vec{v}\right)\left(\hat{n}_{1} \cdot \vec{v}\right)^{2}$. The reason we require that $H_{\xi}(\vec{v})$ is even under the transformation $\vec{v} \rightarrow-\vec{v}$ is as follows. We said above that when general distributions are considered the polarization tensor is obtained from Eqs. (8) and (9) by making the replacement $n(k) \rightarrow n(\vec{k})=C_{\xi} n\left(k H_{\xi}(\vec{v})\right)$. In fact, there is an extra contribution to the anisotropic version of Eq. (8) of the form

$$
\Pi_{\text {extra }}^{i j}\left(p_{0}, \vec{p}\right) \sim g^{2} \int \frac{d^{3} k}{(2 \pi)^{3}}\left[n_{R}(\vec{k})+\bar{n}_{R}(\vec{k})\right]\left(\frac{v^{i} v^{j}}{p_{0}-\vec{p} \cdot \vec{v}+i \epsilon}-\frac{v^{i} v^{j}}{p_{0}+\vec{p} \cdot \vec{v}+i \epsilon}\right)
$$

that integrates to zero for any distribution that is even under the transformation $\vec{k} \rightarrow-\vec{k}$. If the condition $H_{\xi}(\vec{v})=$ $H_{\xi}(-\vec{v})$ is not satisfied, Eq. (17) would produce a nonzero contribution to the polarization tensor that dominates over the HL terms, and is not present in the result obtained from semiclassical kinetic theory (which is equivalent to the HL expression).

We will calculate the anisotropic polarization tensor using Eqs. (11) and (12) with the replacement $n(k) \rightarrow C_{\xi} n\left(k H_{\xi}(\vec{v})\right)$ and similarly for $\bar{n}(k)$. We define $\tilde{k}=k H_{\xi}(\vec{v})$ and perform a straightforward change of variable so that the integral over $\vec{k}$ is written as the product of an integral over the new variable $\tilde{k}$ and a factored integral involving the angular variables. Equations (11) and (12) become

$$
\begin{aligned}
& \Pi_{R \text { even }}^{i j}\left(p_{0}, \vec{p}\right)=\left(m_{D}^{2}\right)_{R} C_{\xi} \int \frac{d \Omega}{4 \pi} \frac{1}{H_{\xi}^{4}(\vec{v})} v^{i} \mathcal{M}^{l}(\Omega)\left(\delta^{j l}+\frac{p^{l} v^{j}}{P \cdot V+i \epsilon}\right), \\
& \Pi_{R \text { odd }}^{i j}\left(p_{0}, \vec{p}\right)=i \frac{g^{2} \mu_{R}}{2 \pi^{2}} \epsilon^{i j m} p C_{\xi} \int \frac{d \Omega}{4 \pi} \frac{1}{H_{\xi}^{3}(\vec{v})} \mathcal{M}^{l}(\Omega)\left(p_{0} \delta^{l m}+\frac{\left(p_{0} v^{m}-p^{m}\right) p^{l}}{P \cdot V+i \epsilon}\right),
\end{aligned}
$$

where we have defined

$$
\mathcal{M}^{l}(\Omega)=\left(\frac{1}{2 k} \frac{\partial\left[k^{2} H_{\xi}^{2}(\vec{v})\right]}{\partial k^{l}}\right)
$$

and used $\left(m_{D}^{2}\right)_{R}=g^{2}\left(T^{2} / 3+\mu_{R}^{2} / \pi^{2}\right)$. The full expression for $\Pi^{i j}$ is obtained using equation (13), as in the isotropic case.

We close this section by explaining how we define the function of the anisotropy parameters which we call $C_{\xi}$, which can be thought of as a normalization of the anisotropic distribution function. This factor is determined by enforcing the condition

$$
\int \frac{d^{3} k}{(2 \pi)^{3}} \frac{n_{\mu=0}(k)}{k} \equiv C_{\xi} \int \frac{d^{3} k}{(2 \pi)^{3}} \frac{1}{k} n_{\mu=0}\left(k H_{\xi}(\vec{v})\right)
$$

from which we obtain

$$
C_{\xi}^{-1}=\int \frac{d \Omega}{4 \pi} \frac{1}{H_{\xi}^{2}(\vec{v})} .
$$

This choice of normalization gives that when the chemical potentials are zero the mass parameter in Eq. (15) is indepen- dent of the anisotropy parameters. At zero chemical potential the entire spectrum of collective modes depends on this one scale [since we have assumed massless partons; see under Eq. (7)], and it is therefore natural to adopt a normalization that leaves the Debye mass invariant under a change of the anisotropy parameters. We also note that using Eqs. (18), (20), and (22) it is easy to see that the even part of the polarization tensor is unchanged when the complete set of anisotropy parameters are scaled uniformly: $\xi_{i} \rightarrow \Lambda \xi_{i}$ where $\Lambda$ is any positive constant. This means that when we compare the spectrum of collective modes obtained from different choices of anisotropy parameters, we are comparing the effects of a specific deformation, and not the influence of an overall change of scale.

\section{B. Projection operators}

To decompose our general expression for the $3 \times 3$ polarization tensor we need a complete set of nine projection operators. This can be done most conveniently using three 
normalized unit vectors that are related to the plasmon momentum $\vec{p}$ and the two anisotropy vectors $\hat{n}_{1}$ and $\hat{n}_{3}$. From these three vectors we construct three orthonormal vectors which we call $\left(\hat{p}, n_{f}, m_{F}\right)$. We define

$$
\begin{aligned}
\hat{p} & =\frac{\vec{p}}{p}, \\
n_{f} & =\frac{\tilde{n}_{f}}{\sqrt{\tilde{n}_{f} \cdot \tilde{n}_{f}}} \text { with } \tilde{n}_{f}=n_{3}-\left(n_{3} \cdot \hat{p}\right) \hat{p}, \\
m_{F} & =\frac{\tilde{m}_{F}}{\sqrt{\tilde{m}_{F} \cdot \tilde{m}_{F}}} \text { with } \tilde{m}_{F}=\tilde{m}_{f}-\left(n_{f} \cdot \tilde{m}_{f}\right) n_{f} \text { and } \tilde{m}_{f}=n_{1}-\left(n_{1} \cdot \hat{p}\right) \hat{p} .
\end{aligned}
$$

Using these unit vectors the projection operators are defined as

$$
\begin{aligned}
& P_{1}^{i j}=m_{F}^{i} m_{F}^{j}, P_{2}^{i j}=\hat{p}^{i} \hat{p}^{j}, P_{3}^{i j}=n_{f}^{i} n_{f}^{j}, \\
& P_{4}^{i j}=\hat{p}^{i} n_{f}^{j}+n_{f}^{i} \hat{p}^{j}, \quad P_{5}^{i j}=\hat{p}^{i} m_{F}^{j}+m_{F}^{i} \hat{p}^{j}, \quad P_{6}^{i j}=n_{f}^{i} m_{F}^{j}+m_{F}^{i} n_{f}^{j}, \\
& P_{7}^{i j}=n_{f}^{i} \hat{p}^{j}-\hat{p}^{i} n_{f}^{j}, \quad P_{8}^{i j}=n_{f}^{i} m_{F}^{j}-m_{F}^{i} n_{f}^{j}, P_{9}^{i j}=\hat{p}^{i} m_{F}^{j}-m_{F}^{i} \hat{p}^{j} .
\end{aligned}
$$

They satisfy conditions of the form $P_{m}^{i j} P_{n}^{j i}=\mathcal{T}_{m n}$, where the $9 \times 9$ tensor $\mathcal{T}$ is

$$
\left(\begin{array}{ccccccccc}
2 P_{1} & 0 & 0 & 0 & P_{5} & P_{6} & 0 & P_{8} & P_{9} \\
0 & 2 P_{2} & 0 & P_{4} & P_{5} & 0 & P_{7} & 0 & P_{9} \\
0 & 0 & 2 P_{3} & P_{4} & 0 & P_{6} & P_{7} & P_{8} & 0 \\
0 & P_{4} & P_{4} & 2\left(P_{2}+P_{3}\right) & P_{6} & P_{5} & 0 & P_{9} & P_{8} \\
P_{5} & P_{5} & 0 & P_{6} & 2\left(P_{1}+P_{2}\right) & P_{4} & P_{8} & P_{7} & 0 \\
P_{6} & 0 & P_{6} & P_{5} & P_{4} & 2\left(P_{1}+P_{3}\right) & -P_{9} & 0 & -P_{7} \\
0 & P_{7} & P_{7} & 0 & P_{8} & -P_{9} & -2\left(P_{2}+P_{3}\right) & -P_{5} & P_{6} \\
P_{8} & 0 & P_{8} & P_{9} & P_{7} & 0 & -P_{5} & -2\left(P_{1}+P_{3}\right) & -P_{4} \\
P_{9} & P_{9} & 0 & P_{8} & 0 & -P_{7} & P_{6} & -P_{4} & -2\left(P_{1}+P_{2}\right)
\end{array}\right) .
$$

The projectors in Eq. (24) form a complete basis, and therefore the polarization tensor can be decomposed as

$$
\Pi^{i j}=\pi_{1} P_{1}^{i j}+\pi_{2} P_{2}^{i j}+\pi_{3} P_{3}^{i j}+\pi_{4} P_{4}^{i j}+\pi_{5} P_{5}^{i j}+\pi_{6} P_{6}^{i j}+i \pi_{7} P_{7}^{i j}+i \pi_{8} P_{8}^{i j}+i \pi_{9} P_{9}^{i j},
$$

where we have omitted the functional arguments to shorten the notation. A decomposition that is related to the first six of our projection operators was defined in Ref. [49]. The last three projection operators are antisymmetric in their indices, and the corresponding dressing functions can only be nonzero if the chiral chemical potential is nonzero. We invert the Dyson equation (1) using (25) and obtain the propagator

$$
\begin{aligned}
\mathcal{D} D^{i j}= & {\left[\left(p_{0}^{2}-\pi_{2}\right)\left(P^{2}-\pi_{3}\right)-\pi_{4}^{2}-\pi_{7}^{2}\right] P_{1}^{i j}+\left[\left(P^{2}-\pi_{1}\right)\left(P^{2}-\pi_{3}\right)-\pi_{6}^{2}-\pi_{8}^{2}\right] P_{2}^{i j}+\left[\left(p_{0}^{2}-\pi_{2}\right)\left(P^{2}-\pi_{1}\right)-\pi_{5}^{2}-\pi_{9}^{2}\right] P_{3}^{i j} } \\
& +\left[\left(P^{2}-\pi_{1}\right) \pi_{4}+\pi_{5} \pi_{6}+\pi_{8} \pi_{9}\right] P_{4}^{i j}+\left[\left(P^{2}-\pi_{3}\right) \pi_{5}+\pi_{4} \pi_{6}+\pi_{7} \pi_{8}\right] P_{5}^{i j}+\left[\left(p_{0}^{2}-\pi_{2}\right) \pi_{6}+\pi_{4} \pi_{5}-\pi_{7} \pi_{9}\right] P_{6}^{i j} \\
& +i\left[\left(P^{2}-\pi_{1}\right) \pi_{7}+\pi_{5} \pi_{8}-\pi_{6} \pi_{9}\right] P_{7}^{i j}+i\left[\left(p_{0}^{2}-\pi_{2}\right) \pi_{8}+\pi_{5} \pi_{7}+\pi_{4} \pi_{9}\right] P_{8}^{i j}+i\left[\left(P^{2}-\pi_{3}\right) \pi_{9}-\pi_{6} \pi_{7}+\pi_{4} \pi_{8}\right] P_{9}^{i j}, \quad(27)
\end{aligned}
$$

where we have defined

$$
\begin{aligned}
\mathcal{D}= & \left(p_{0}^{2}-\pi_{2}\right)\left(P^{2}-\pi_{1}\right)\left(P^{2}-\pi_{3}\right)-\left(P^{2}-\pi_{1}\right)\left(\pi_{4}^{2}+\pi_{7}^{2}\right)-\left(p_{0}^{2}-\pi_{2}\right)\left(\pi_{6}^{2}+\pi_{8}^{2}\right) \\
& -\left(P^{2}-\pi_{3}\right)\left(\pi_{5}^{2}+\pi_{9}^{2}\right)-2\left(\pi_{4} \pi_{5} \pi_{6}-\pi_{7} \pi_{9} \pi_{6}+\pi_{5} \pi_{7} \pi_{8}+\pi_{4} \pi_{8} \pi_{9}\right) .
\end{aligned}
$$

The anisotropic dispersion relations are the solutions of the dispersion equation, $\mathcal{D}=0$.

\section{Coordinate system}

To make further process we must define a coordinate system. The vector along the beam axis can be chosen as $\hat{n}_{3}^{\prime}=(0,0,1)$, and the vector that defines the direction of asymmetry in the transverse plane can then be chosen as $\hat{n}_{1}^{\prime}=$ $(1,0,0)$. The external momentum vector then has the general form $\vec{p}^{\prime}=p(\sin \theta \cos \phi, \sin \theta \sin \phi, \cos \theta)$. We note that using these coordinates it is easy to see that the parametrization in Eq. (16) is equivalent to an expansion in spherical harmonics $Y_{l m}(\theta, \phi)$ with $l \in(0,4)$ and $m \in(-l, l)$.

We can define coordinates that are more convenient for calculational purposes by performing a rotation [12]. We rotate counterclockwise about the $z$ axis with angle $\theta_{z}=\pi / 2-\phi$ and then counterclockwise about the $x$ axis with $\theta_{x}=\theta$. The result is that the basis vectors become

$$
\begin{aligned}
& \hat{n}_{1}=(\sin (\phi), \cos (\theta) \cos (\phi), \sin (\theta) \cos (\phi)), \\
& \hat{n}_{3}=(0,-\sin (\theta), \cos (\theta)), \\
& \hat{p}=\vec{p} / p=(0,0,1)
\end{aligned}
$$


TABLE I. Different sets of anisotropy parameters, the corresponding values of the parameters $\xi$, $\varepsilon$, and $v_{2}$ defined in Eq. (31), the size and location of the largest imaginary solution, and the integral of the solution over its domain. In each case the anisotropy parameters that are not specified are set to zero.

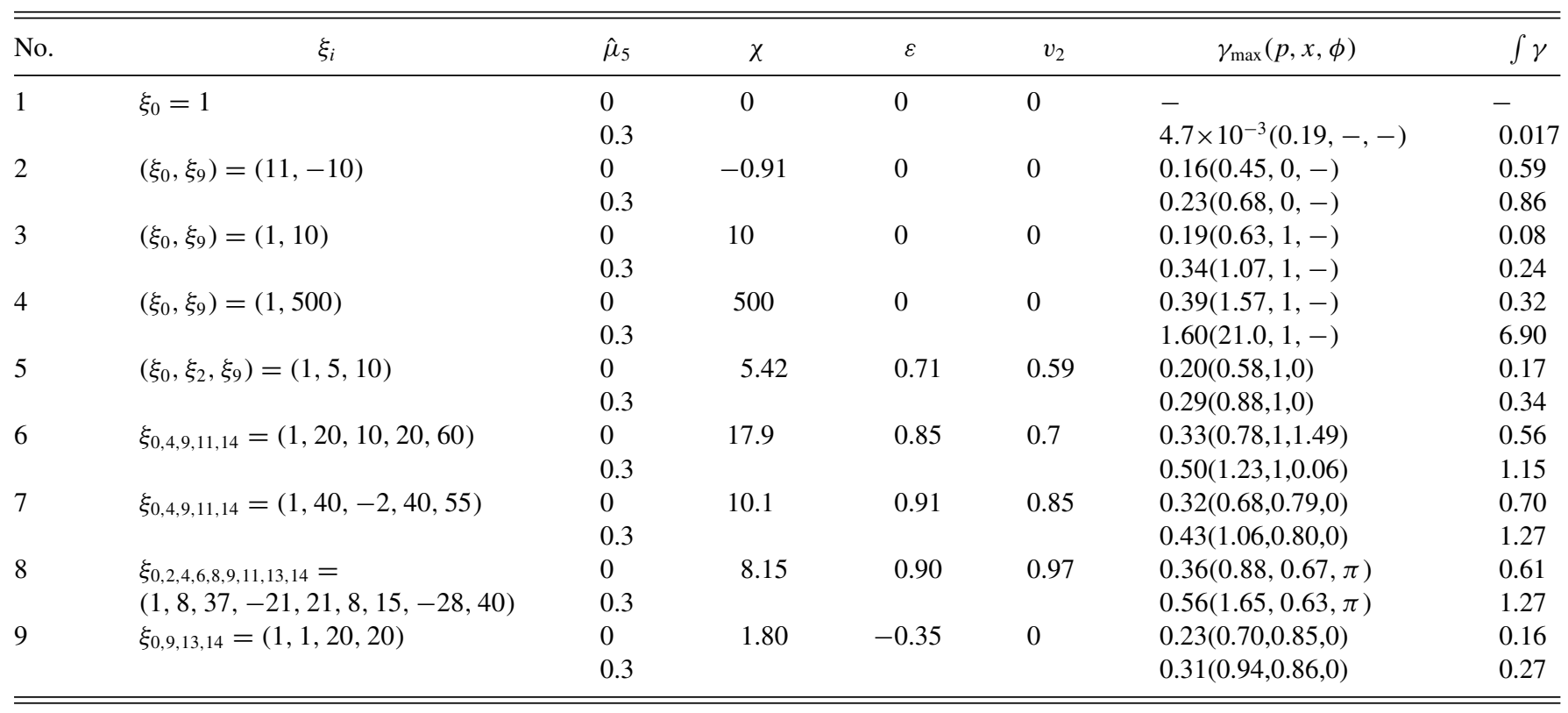

Any scalar or pseudoscalar quantity will give the same result when calculated with either the original or the rotated coordinate system. This can be easily seen from the fact that the scalar products $\left(\hat{p} \cdot \hat{n}_{1}\right),\left(\hat{p} \cdot \hat{n}_{3}\right)$, and $\left(\hat{n}_{1} \cdot \hat{n}_{3}\right)$, and the pseudoscalar $\epsilon^{i j k} \hat{n}_{1}^{i} \hat{n}_{3}^{j} \hat{p}^{k}$, are invariant. Likewise the dispersion relations are the same in either coordinate system. Another useful thing about the coordinates in Eq. (29) is that the isotropic limit has a simple form. It is easy to show using Eq. (29) that $\delta^{i j}-\hat{p}^{i} \hat{p}^{j}=P_{1}^{i j}+P_{3}^{i j}, \hat{p}^{i} \hat{p}^{j}=P_{2}^{i j}$, and $\epsilon^{i j m} p^{m}=P_{8}^{i j}$. In the isotropic limit there are only three different nonzero components in Eq. (26) which are $\pi_{1}=\pi_{3}=\Pi_{T}$, $\pi_{2}=\Pi_{L}$, and $\pi_{8}=\Pi_{A}$. Using these results one can see that Eqs. (27) and (28) reduce to (3).

The variables in the angular integrations in Eqs. (18) and (19) are independent variables, and will be written in component form as

$$
\vec{v}=\left(\sin \left(\theta^{\prime}\right) \cos \left(\phi^{\prime}\right), \sin \left(\theta^{\prime}\right) \sin \left(\phi^{\prime}\right), \cos \left(\theta^{\prime}\right)\right) .
$$

We will use the notation $x=\cos (\theta)$ and $x^{\prime}=\cos \left(\theta^{\prime}\right)$.

\section{Anisotropy parameters}

The anisotropy parameters $\xi_{9}$ and $\xi_{2}$ produce uniform stretching or squeezing of the isotropic distribution along the beam axis (in the case of $\xi_{9}$ ), and along the vector $\hat{n}_{1}$ in the transverse plane (for $\xi_{2}$ ). We have introduced additional anisotropy parameters that can be used to produce distribution functions that are deformed relative to the isotropic distribution in more general ways, and produce dispersion relations with more structure. As discussed in Sec. III A, we consider only distributions that satisfy the condition $f(\vec{k})=$ $f(-\vec{k})$. This restricts the form of the terms in Eq. (16). Each term is chosen so that $e_{1}+e_{3}$ is even, where the exponent of the factor $\left(\vec{n}_{1} \cdot \vec{v}\right)$ is denoted $e_{1}$ and the exponent of the factor $\left(\vec{n}_{3} \cdot \vec{v}\right)$ is called $e_{3}$. There are two different possibilities: $e_{1}$ and $e_{3}$ individually even, or $e_{1}$ and $e_{3}$ individually odd.

The elliptically anisotropic distribution considered in [12] depends only on the two parameters $\xi_{9}$ and $\xi_{2}$, both of which have $e_{1}$ and $e_{3}$ individually even. Any distribution for which $e_{1}$ and $e_{3}$ are individually even for all terms in (16) can be mapped onto an elliptically anisotropic distribution with angularly dependent parameters. We consider for example the distribution constructed with the set of anisotropy parameters $\xi_{0}=1, \xi_{4}=20, \xi_{9}=10, \xi_{11}=20, \xi_{14}=60$ (distribution 6 in Table I). This distribution is equivalent to an elliptically anisotropic distribution with effective coefficients $\xi_{2}(\Omega)$ and $\xi_{9}(\Omega)$ as shown in Fig. 2. The new parameters $\xi_{4}, \xi_{11}$, and $\xi_{14}$ effectively modify the constant value of $\xi_{9}$ and generate a nonzero $\xi_{2}$.

The parameters $\xi_{6}, \xi_{8}$, and $\xi_{13}$ are coefficients of terms in Eq. (16) with $e_{1}$ and $e_{3}$ individually odd. These terms produce a distortion of the isotropic distribution that is qualitatively different. Some examples of the dispersion relations obtained from distributions with nonzero values of these coefficients are presented in Sec. IV.

To illustrate more directly the roles of the different anisotropy parameters we look at plots of $C_{\xi} n\left(k H_{\xi}(\vec{v})\right)$ with $\beta=1$ and $\mu=0$, in the $\left(p_{x}, p_{y}\right)$ and $\left(p_{x}, p_{z}\right)$ planes. In Fig. 3 we show the results for several choices of anisotropy parameters. The first panel shows, for reference, the isotropic distribution in the $\left(p_{x}, p_{y}\right)$ plane; the figure for the $\left(p_{x}, p_{z}\right)$ plane is identical. In the second panel we show a prolate distribution in the $\left(p_{x}, p_{z}\right)$ plane, and one sees clearly that the distribution is stretched along the beam axis. The third panel shows the squeezing of an oblate distribution along the beam axis. In the fourth and fifth panels we show an elliptically oblate distribution, which is asymmetric in both planes. In the sixth panel we show an example of the kind of structure that 

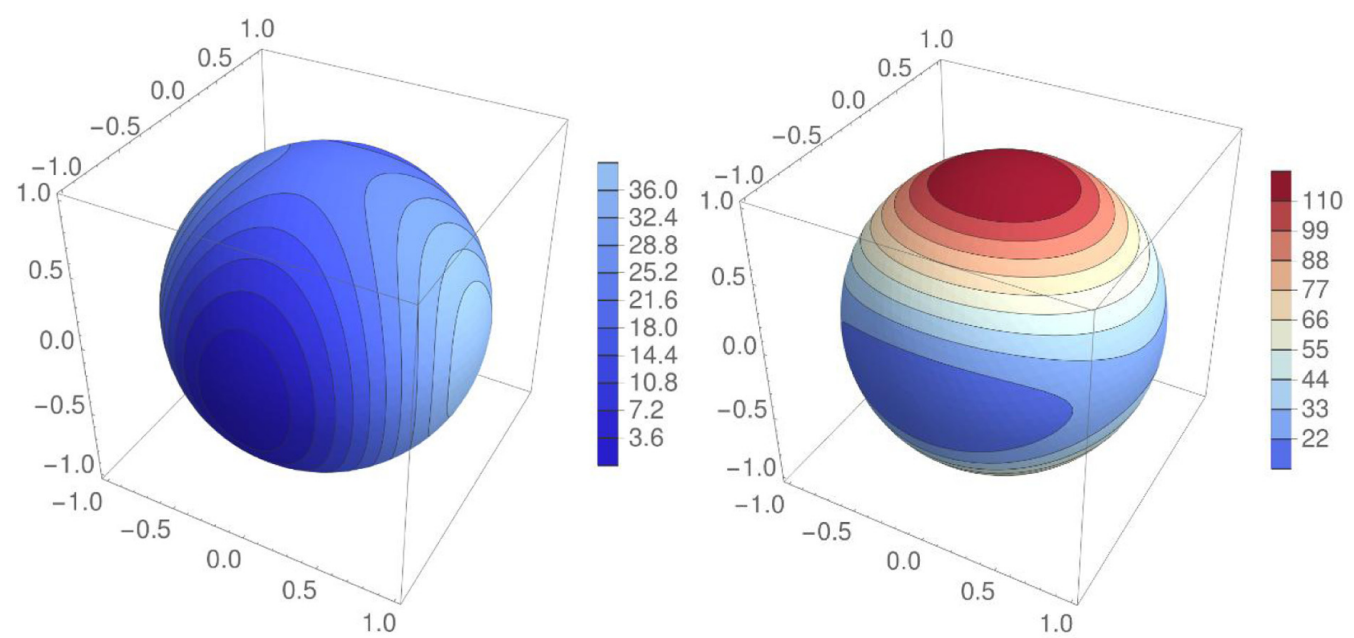

FIG. 2. Spherical plots of the coefficients $\xi_{2}(\Omega)$ (left panel) and $\xi_{9}(\Omega)$ (right panel) that correspond to the mapping of distribution 6 in Table I onto an elliptically anisotropic distribution. The values in the figure are obtained with $\beta=1$ and $\mu=0$.

can be obtained with a specific choice of the extra anisotropy parameters we have introduced.

\section{DISPERSIONS RELATIONS}

\section{A. Analytic structure}

The isotropic and anisotropic dressing functions have the same analytic structure, which can be determined from Eqs. (11) and (12), using that Eqs. (29) and (30) give $P$. $V=p\left(\hat{p}_{0}-x^{\prime}\right)$, where $\hat{p}_{0}=p_{0} / p$. When $\hat{p}_{0}$ is pure real the epsilon prescription is needed to define the integrands at $\hat{p}_{0}=x^{\prime}$. Shifting the integration variable $\vec{v} \rightarrow-\vec{v}$ one shows that $\pi_{i}^{*}\left(\hat{p}_{0}\right)=\pi_{i}\left(-\hat{p}_{0}\right)$, where the subscript $i$ indicates any component of the polarization tensor. This means that when $\hat{p}_{0}$ is real, the real part of each dressing function is even in $\hat{p}_{0}$ and the imaginary part is odd. We also note that all dressing functions are pure real for $\hat{p}_{0}>1$, since the imaginary part comes from the discontinuity at $\hat{p}_{0}=x^{\prime}$ and $\left|x^{\prime}\right|<1$. When $\hat{p}_{0}$ is not pure real the epsilon prescription is not needed, and we have $\pi_{i}^{*}\left(\hat{p}_{0}\right)=\pi_{i}\left(\hat{p}_{0}^{*}\right)$. When $p_{0}$ is pure imaginary $\pi_{i}\left(\hat{p}_{0}^{*}\right)=\pi_{i}\left(\hat{p}_{0}\right)$, which can be shown by shifting the integration variable, and therefore the dressing functions are pure real and even in $\hat{p}_{0}$. The information above can be summarized as

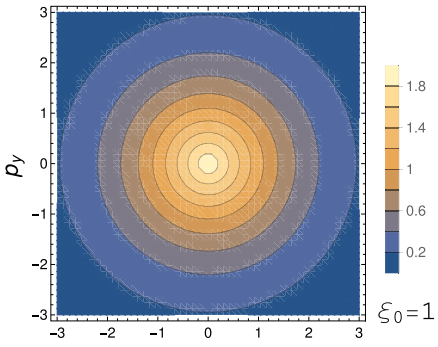

$p_{x}$

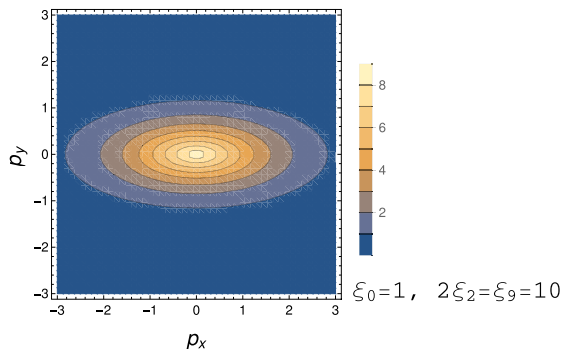

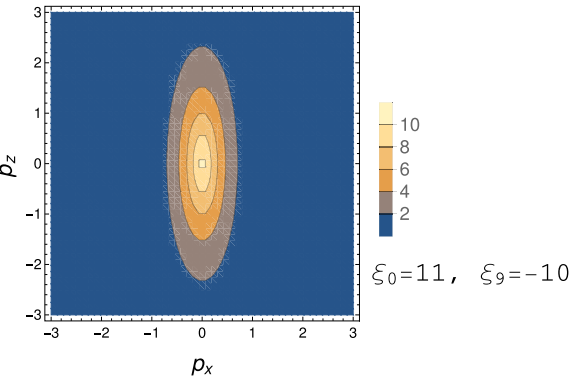

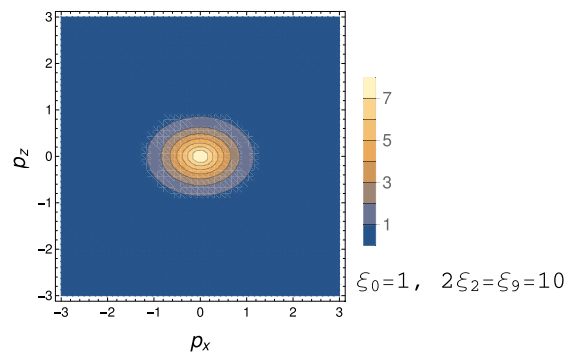

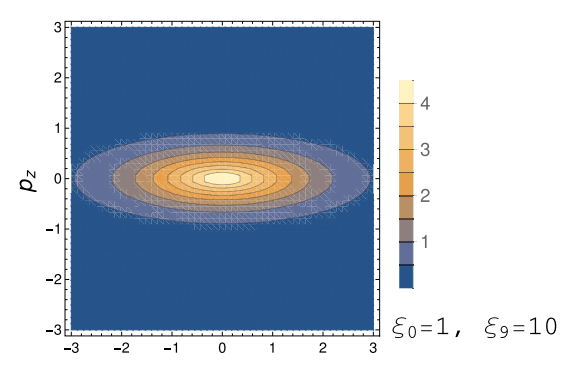

$p_{x}$

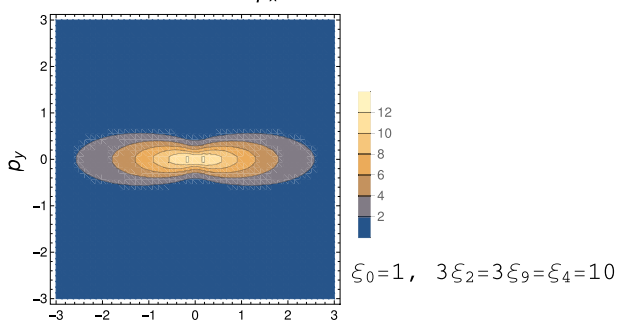

$p_{x}$

FIG. 3. Contour plots of the distribution for different choices of the anisotropy parameters. In each case the parameters that are not specified are set to zero. 
$\omega$
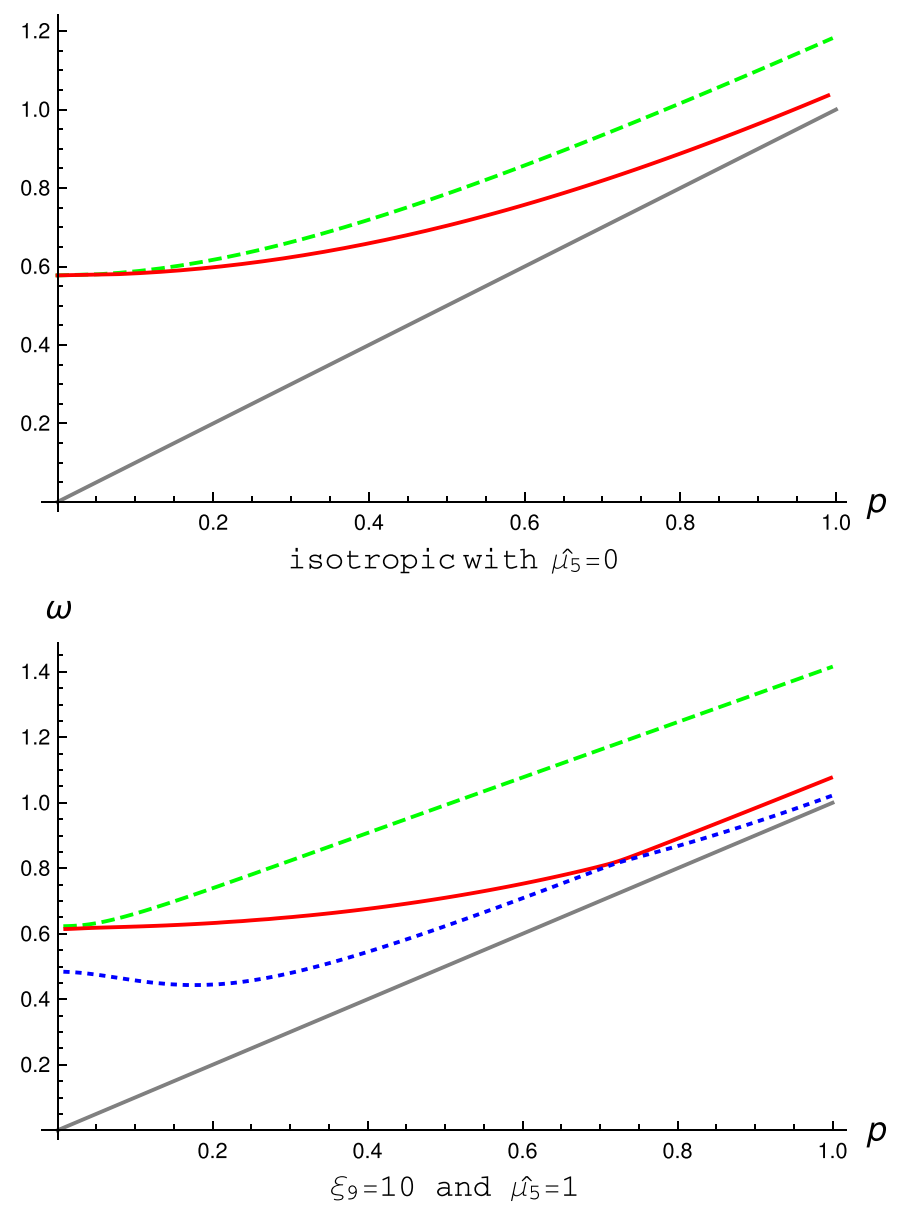

$\omega$
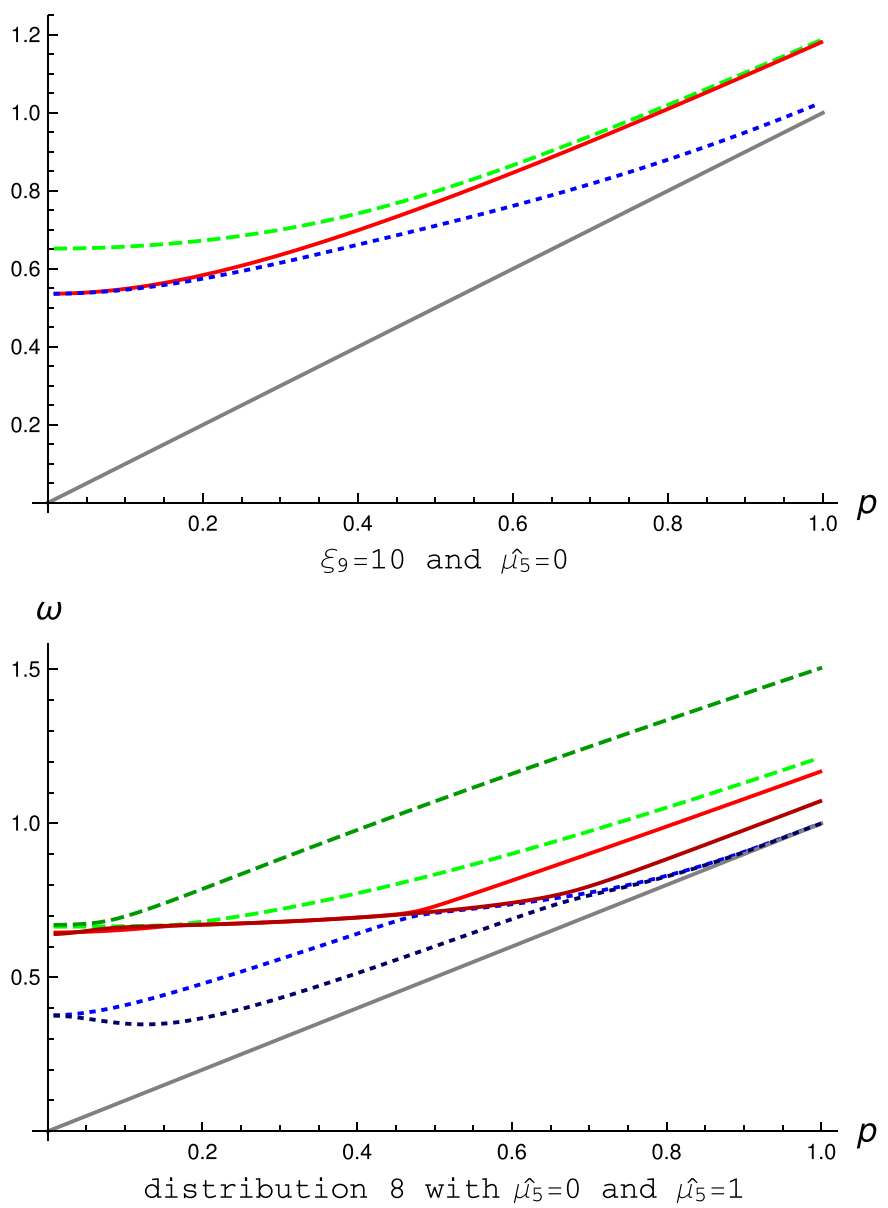

FIG. 4. Real solutions for distributions 1,3 , and 8 (as defined in Table I). In the bottom right panel the lighter lines are the solutions with $\hat{\mu}_{5}=0$ and the dark lines are $\hat{\mu}_{5}=1$. In all graphs the light gray line shows the light cone.

\section{B. Method}

The integrand for the polarization tensor is given by Eqs. (18)-(20). The nine scalar components $\pi_{1}-\pi_{9}$ are obtained by contracting with the appropriate projection operators, which are easily obtained from Eqs. (25) and (26). These calculations are straightforward but tedious, and they are therefore done with MATHEMATICA. The resulting integrals are calculated numerically using Gauss-Legendre quadrature. For real-valued $\hat{p}_{0}$, the real part of the dressing functions has a single integrable singularity at $x^{\prime}=\hat{p}_{0}$ that is easily handled with a numerical principal part prescription, and the imaginary part can be obtained from the residue of the pole. For imaginary valued $\hat{p}_{0}$ there are no difficulties. After the dressing functions are obtained, the dispersion equation is solved with a standard binary search algorithm.

\section{Real solutions}

For any choice of the anisotropy parameters and the chiral chemical potential $\mu_{5}$ there are three real solutions. We show some examples of the dispersion relations in Fig. 4.

\section{Imaginary solutions}

We are primarily interested in imaginary solutions, because of their potential to influence plasma dynamics. We will compare the imaginary modes produced by different choices of the anisotropy parameters, and explore their dependence on these parameters as a function of wave vector. We have previously used the notation $\gamma(\vec{p})$ to indicate the dependence of the dispersion relation on the wave vector, but in this section we will use the more explicit notation $\gamma(p, x, \phi)$, where $x=\cos (\theta)$. For different sets of anisotropy parameters we compare the maximum value of the imaginary solution, the position of this maximum, and the integral of the solution over its domain, which we write $\int \gamma \equiv \int_{0}^{\infty} d p \int_{-1}^{1} d x \int_{0}^{2 \pi} d \phi \gamma(p, x, \phi)$.

We define three variables that characterize the degree of oblateness, the transverse eccentricity, and the azimuthal asymmetry of a given distribution:

$$
\begin{aligned}
& \chi=\frac{\left\langle k_{x}^{2}\right\rangle+\left\langle k_{y}^{2}\right\rangle}{2\left\langle k_{z}^{2}\right\rangle}-1, \quad \varepsilon=\frac{\left\langle k_{y}^{2}\right\rangle-\left\langle k_{x}^{2}\right\rangle}{\left\langle k_{y}^{2}\right\rangle+\left\langle k_{x}^{2}\right\rangle}, \\
& v_{2}=\frac{\int d k k^{2} \int d \phi \cos (2 \phi) n\left(k H_{\xi}(\vec{v})\right)_{x=0}}{\int d k k^{2} \int d \phi n\left(k H_{\xi}(\vec{v})\right)_{x=0}} .
\end{aligned}
$$



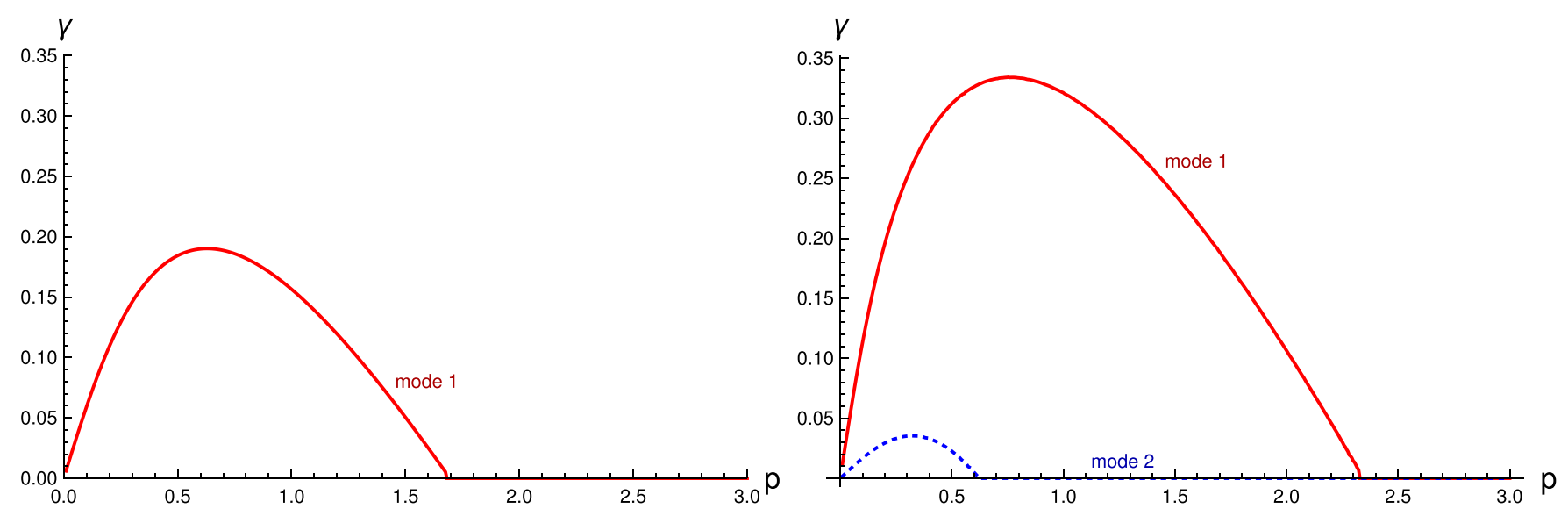

FIG. 5. The imaginary solutions of the dispersion equation $\mathcal{D}=0$ obtained from distribution 3 (left panel) with $\hat{\mu}_{5}=0$ and $x=1$, and the two imaginary solutions obtained from distribution 6 (right panel) with $\hat{\mu}_{5}=0, x=1$, and $\phi=\pi / 2$.

The angle brackets denote averaging over the momentum phase space, for example $\left\langle k_{x}^{2}\right\rangle=\int d^{3} k k_{x}^{2} C_{\xi} n\left(k H_{\xi}(\vec{v})\right)$.

In Table I we show some of the main features of the largest imaginary solution of the dispersion relation for several different distributions. In all cases the anisotropy parameters that are not specified are zero. The first distribution is the isotropic one; the second and third have spheroidal anisotropy, with parameters that make them prolate and oblate, respectively. The fourth is a distribution with spheroidal anisotropy that is strongly oblate. The fifth is an elliptically anisotropic distribution. Distributions 6-9 are examples constructed using the additional anisotropy parameters that we have introduced. Distributions 6 and 7 involve only terms for which $e_{1}$ and $e_{3}$ are even (see the discussion at the beginning of Sec. IIID). The second to last column shows that the maximum of the largest mode can be much greater than the result obtained from a distribution with spheroidal anisotropy with a comparable value of the oblateness parameter $\chi$. In addition, the last column shows that the integral of the solution over its full domain is greatly enhanced, relative to the result obtained from a comparable distribution with spheroid oblateness. For example, distribution 7 has approximately the same value of $\chi$ as distribution 3 , but $\gamma_{\max }$ is about twice as big, and the integral of the solution is almost 9 times larger. Distributions 8 and 9 are examples for which $e_{1}$ and $e_{3}$ are individually odd for some of the terms in (16). The magnitudes of the modes produced by these distributions are similar to what is obtained from distributions without these odd coefficients, but the angular dependence of the solutions is much more complex. At the end of this section we show several plots to demonstrate this. Finally, the table shows that the effect of a nonzero chiral chemical potential is always to increase the magnitude and domain of the imaginary solution. The solutions produced with even moderate anisotropy are much larger than those obtained with the same chemical potential in an isotropic system.

For a given distribution, the number of imaginary solutions is 0 or 1 or 2 , depending on the magnitude and direction of the wave vector. For each mode there is a critical vector $p_{\text {crit }}\left(x, \phi, \xi_{i}\right)$ such that the imaginary mode appears for $p$ less than this critical value. Analytic results for the critical wave vectors can be obtained in the limit of weak anisotropy using a Nyquist analysis. The method is explained in detail in Appendix B, where we show how to identify the angular variables that produce the largest critical wave vectors for a given set of anisotropy parameters. To understand the structure of the solutions obtained from a given distribution in more detail, we show several figures below.

In Fig. 5 we compare the imaginary solutions obtained from the distributions 3 and 6 for a specific choice of angles. The figure illustrates the concept of the critical wave vector. For example, in the right panel of Fig. 5, the critical wave vectors for modes 1 and 2 are, respectively, 2.33 and 0.62 . It is true in general that when the domain of the solution increases, the maximum value of the solution also increases, as seen in Fig. 5.

In Fig. 6 we show the largest imaginary mode obtained from distribution 6 for different choices of angles, and two different values of the chiral chemical potential. The figure shows that increasing the chiral chemical potential increases the domain and size of the solution, but not uniformly as a function of the angular variables.

The dependence of the imaginary modes on the angular variables can be seen most clearly using contour plots. In the top left panel of Fig. 7 we show a contour plot of the magnitude of the largest imaginary solution for distribution 3 with $\hat{\mu}_{5}=0$, as a function of $p$ and $x$ (there is no $\phi$ dependence). The solution is nonzero in a small region of the phase space where $x$ is close to 1 and $p$ is small. In the top right panel of Fig. 7 we show the largest imaginary solution for distribution 2 with $\hat{\mu}_{5}=0$, which is also $\phi$ independent. The magnitude of the solution is smaller, but the domain is larger. The bottom two panels show the $\phi$ dependent solutions obtained from distribution 5 .

The distributions 6-9 involve anisotropy parameters that correspond to higher spherical harmonics, and have a much richer structure. In Figs. 8 and 9 we show some contour plots of the largest imaginary solution obtained from these distributions with $\hat{\mu}_{5}=0$, for different choices of $x=\cos (\theta)$ and $\phi$.

The effect of the anisotropy parameters which correspond to terms with $e_{1}$ and $e_{3}$ individually odd (see the discussion 


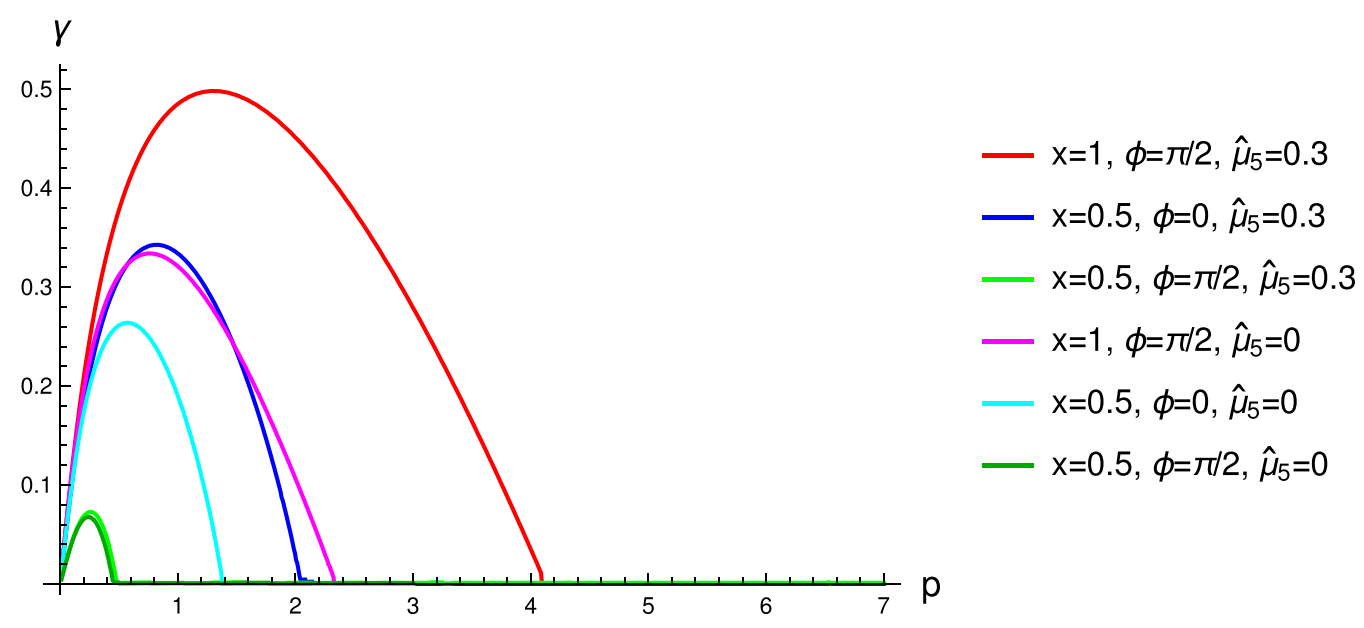

FIG. 6. The largest imaginary solution of the dispersion equation $\mathcal{D}=0$ using the distribution 6 , two different chiral chemical potentials, and several different values of the angular variables.

at the beginning of Sec. IIID) can be seen by calculating $s_{2} \equiv \int_{0}^{\infty} d p \int_{0}^{1} d x \int_{0}^{\pi} d \phi \sin (2 \phi) \gamma(p, x, \phi)$. Any distribution for which $\xi_{6}=\xi_{8}=\xi_{13}=0$ will give $s_{2}=0$, which corresponds physically to symmetry across the reaction plane. If any of these anisotropy parameters are nonzero, the value of $s_{2}$ will also be nonzero. Physically this effect could be produced by a collision at nonzero impact parameter of two ions of different size. Distribution 6 and 7 , for which we should find $s_{2}=0$, give $s_{2} \approx 9 \times 10^{-10}$ and $s_{2} \approx 7 \times 10^{-8}$, which can be taken as a measure of the numerical error in our computation. Distributions 8 and 9 give, respectively, $s_{2}=-0.059$ and $s_{2}=0.068$.

Finally, to show the effect of $\hat{\mu}_{5} \neq 0$, we plot in Fig. 10 the solutions obtained with $\hat{\mu}_{5}=0.3$ using the distributions 6
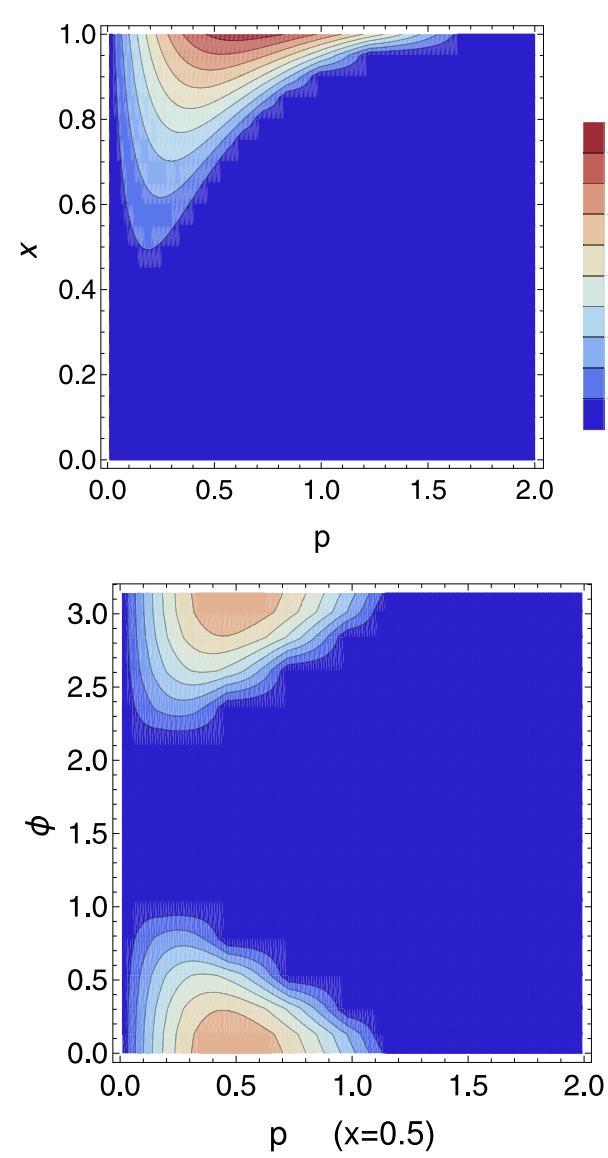
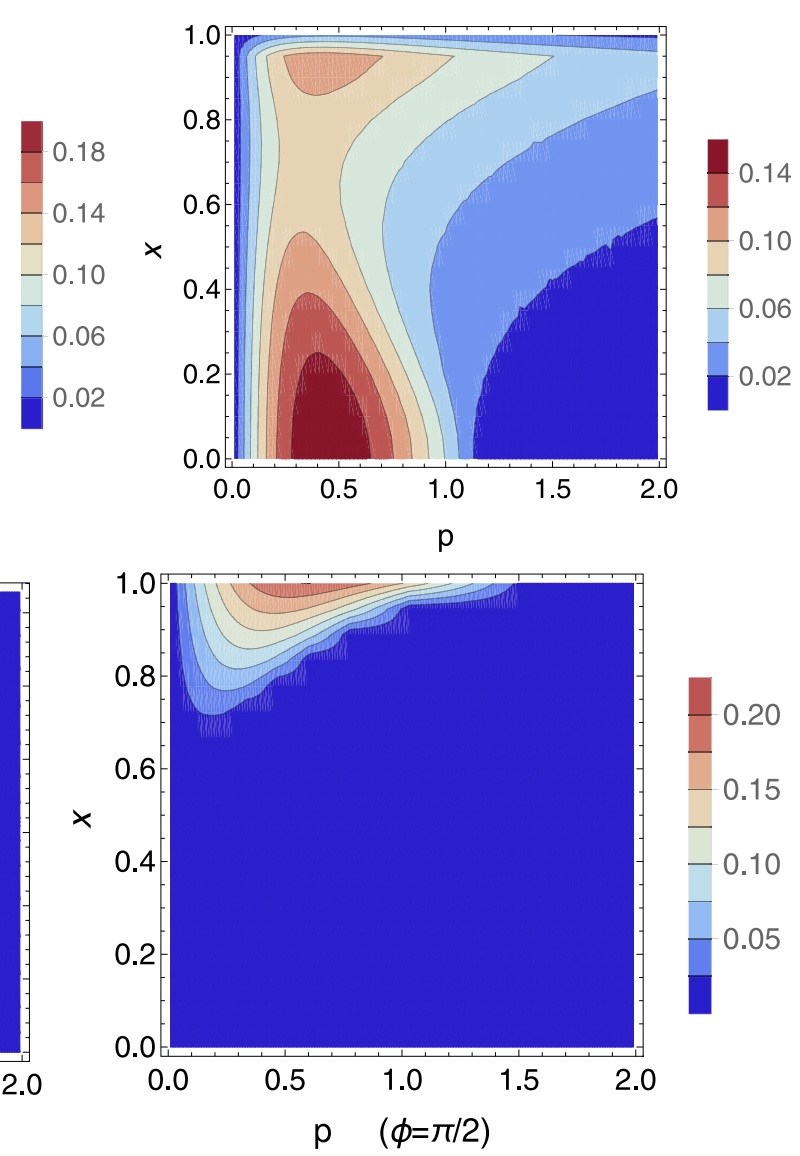

FIG. 7. Contour plots of the largest imaginary solution for several distributions with $\hat{\mu}_{5}=0$. The top left and top right panels show the $\phi$ independent solution obtained from distributions 3 and 2, respectively. The bottom panels are solutions obtained from distribution 5. 

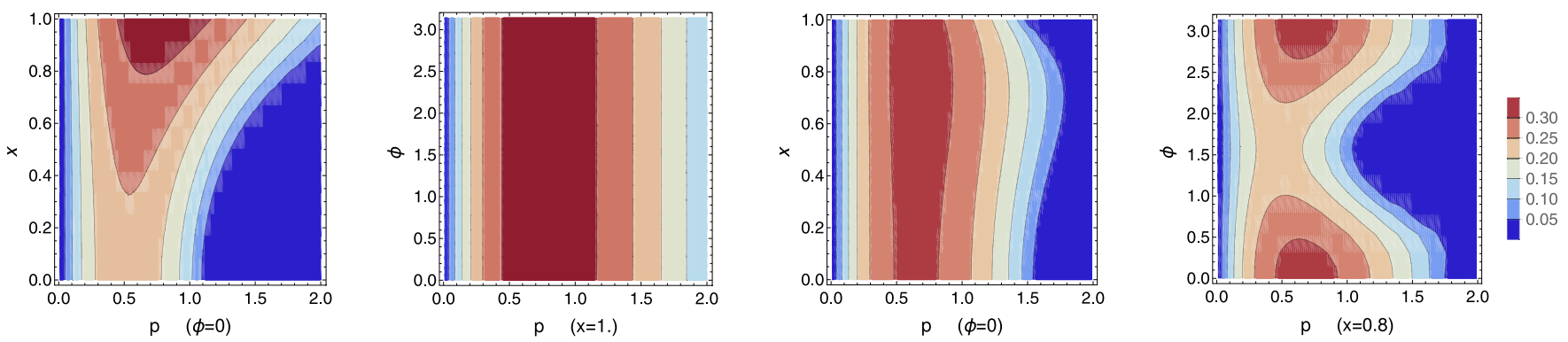

FIG. 8. Contour plots of the largest imaginary solution from the distributions 6 (left two panels) and 7 (right two panels) with $\hat{\mu}_{5}=0$.

and 7. Comparison with Fig. 8 shows that the chiral chemical potential increases the size and domain of the imaginary solution, but not uniformly.

\section{CONCLUSIONS}

We have studied plasmons in anisotropic plasmas using a hard-loop approach within the quasiparticle regime. We have used more general distribution functions than have been previously considered, with a technique that could be easily generalized to account for the anisotropies that are relevant in any given physical situation. The distribution function we have used depends on 9 anisotropy parameters and is normalized so that at zero chemical potential the Debye mass parameter is the same for all distributions. In an ultrarelativistic plasma at zero chemical potential this parameter is the only dimensionful scale. It is therefore meaningful to compare the solutions obtained from the dispersion equations for different choices of anisotropy parameters.

Imaginary solutions to the dispersion equation are important because they are associated with plasma instabilities, which have the potential to strongly influence plasma dynamics. The important effect of imaginary collective modes on physical quantities in anisotropic plasmas, like transport coefficients, production rates, and bound state properties, is well documented in the literature. We have shown that the more general distribution functions we have introduced can significantly increase imaginary solutions of the dispersion equation in terms of both the magnitude of the mode, and the domain of wave vectors over which it exists. The distribution that is most commonly used in the literature depends on only one anisotropy parameter, which describes the oblateness of a distribution that is squeezed in one direction. An example is our distribution 3. In comparison, our distribution 7 , which has approximately the same value of the parameter $\xi$ that characterizes its oblateness (see Table I), produces a largest imaginary mode that is almost twice as big, and the integral of the solution over the momentum phase space is approximately 10 times larger. We have also shown that the effect of the chiral chemical potential is greatly enhanced by anisotropy. This is seen clearly from the results in Table I which show that, relative to the isotropic result, the imaginary modes are much greater when even very moderate anisotropy is introduced.

\section{ACKNOWLEDGMENT}

This work was supported by the Natural Sciences and Engineering Research Council of Canada Discovery Grant program, Grant No. 2017-00028.

\section{APPENDIX A: RETARDED POLARIZATION TENSOR}

In this Appendix we calculate the one-loop retarded polarization tensor at finite temperature and chemical potential with the real-time formulation of finite-temperature field theory, using the Keldysh representation [50-52].

The electron propagator is a $2 \times 2$ matrix of the form

$$
G=\left(\begin{array}{ll}
G_{r r} & G_{r a} \\
G_{a r} & G_{a a}
\end{array}\right)=\left(\begin{array}{cc}
G_{\mathrm{sym}} & G_{\mathrm{ret}} \\
G_{\mathrm{adv}} & 0
\end{array}\right),
$$

where the retarded, advanced, and symmetric propagators are given by

$$
\begin{aligned}
& G_{\mathrm{ret}}\left(p_{0}, p\right)=(\not P+m) r\left(p_{0}, p\right), \\
& G_{\mathrm{adv}}\left(p_{0}, p\right)=(\not P+m) a\left(p_{0}, p\right), \\
& G_{\mathrm{sym}}\left(p_{0}, p\right)=(\not P+m) f\left(p_{0}, p\right),
\end{aligned}
$$
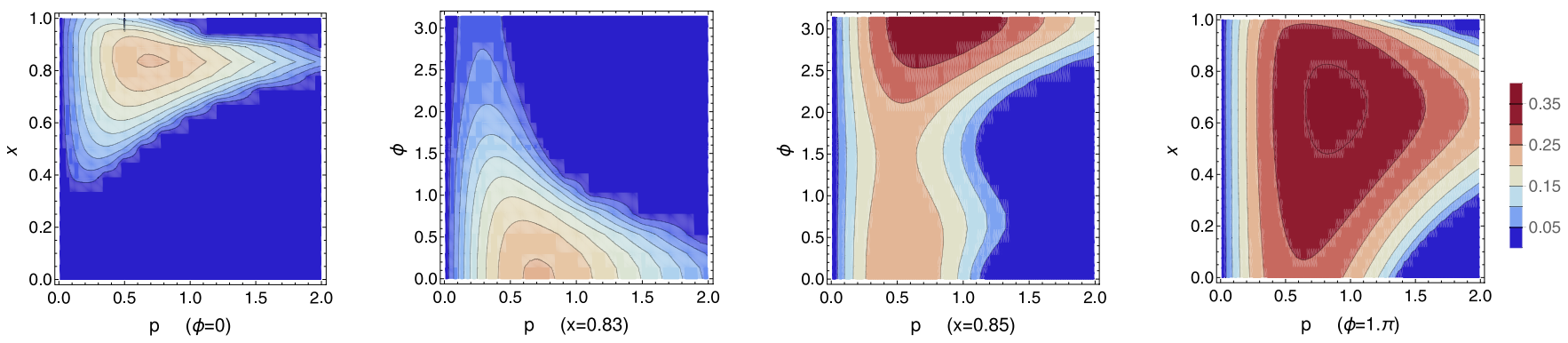

FIG. 9. Contour plots of the largest imaginary solution from the distributions 9 (left two panels) and 8 (right two panels) with $\hat{\mu}_{5}=0$. 

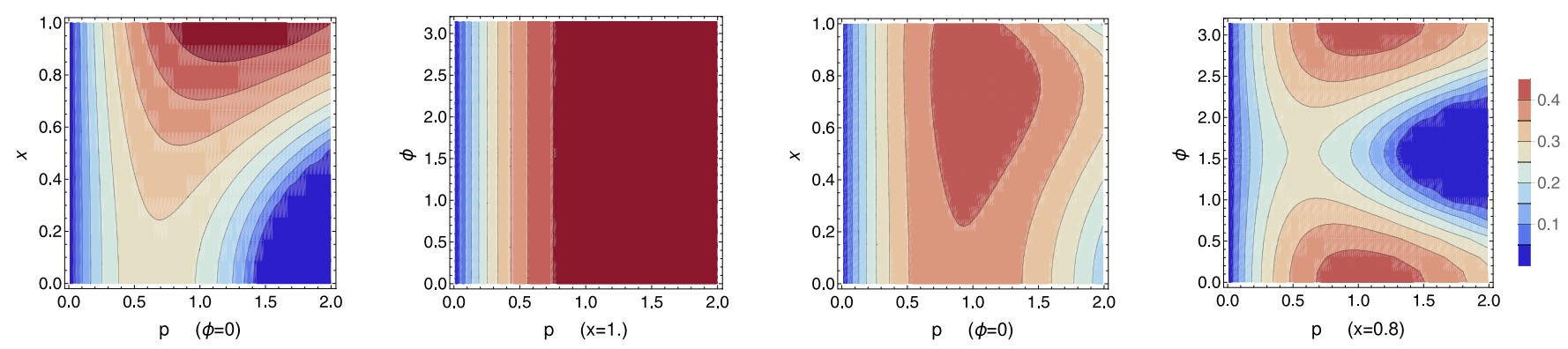

FIG. 10. Contour plots of the largest imaginary solution for the distributions 6 (left two panels) and 7 (right two panels) with $\hat{\mu}_{5}=0.3$.

with

$$
\begin{aligned}
r\left(p_{0}, p\right) & \equiv \frac{1}{P^{2}-m^{2}+i 0^{+} \operatorname{sgn}\left(p_{0}\right)}, \\
a\left(p_{0}, p\right) & \equiv \frac{1}{P^{2}-m^{2}-i 0^{+} \operatorname{sgn}\left(p_{0}\right)}, \\
f\left(p_{0}, p\right) & \equiv-2 \pi i\left\{\left[1-2 n^{+}\left(p_{0}\right)\right] \Theta\left(p_{0}\right)+\left[1-2 n^{-}\left(p_{0}\right)\right] \Theta\left(-p_{0}\right)\right\} \delta\left(P^{2}-m^{2}\right),
\end{aligned}
$$

where

$$
n^{+}\left(p_{0}\right)=\frac{1}{e^{\beta\left(p_{0}-\mu\right)}+1} \quad \text { and } \quad n^{-}\left(p_{0}\right)=\frac{1}{e^{-\beta\left(p_{0}-\mu\right)}+1}
$$

The self-energy has the form

$$
\Pi=\left(\begin{array}{ll}
\Pi_{r r} & \Pi_{r a} \\
\Pi_{a r} & \Pi_{a a}
\end{array}\right)=\left(\begin{array}{cc}
0 & \Pi_{\mathrm{adv}} \\
\Pi_{\mathrm{ret}} & \Pi_{\mathrm{sym}}
\end{array}\right)
$$

and the vertex function is a $2 \times 2 \times 2$ tensor which can be written

$$
\Gamma^{\mu}=-i e \gamma^{\mu}\left(\begin{array}{ll}
\left\{\Gamma_{r r r}, \Gamma_{r r a}\right\} & \left\{\Gamma_{\text {rar }}, \Gamma_{\text {raa }}\right\} \\
\left\{\Gamma_{\text {arr }}, \Gamma_{\text {ara }}\right\} & \left\{\Gamma_{\text {aar }}, \Gamma_{\text {aaa }}\right\}
\end{array}\right)=-i e \gamma^{\mu}\left(\begin{array}{ll}
\{0,1\} & \{1,0\} \\
\{1,0\} & \{0,1\}
\end{array}\right) .
$$

The contribution to the retarded self-energy from the one-loop diagram is

$$
i \Pi_{\mathrm{ret}}^{\mu v}\left(p_{0}, p\right)=i \Pi_{a r}^{\mu \nu}\left(p_{0}, p\right)=\frac{(-i e)^{2}}{2} \sum_{i i^{\prime} j j^{\prime}} \int \frac{d^{4} k}{(2 \pi)^{4}} \gamma^{\mu} \Gamma_{a i j} G_{j j^{\prime}}(k) G_{i^{\prime} i}(k-p) \gamma^{\nu} \Gamma_{r i^{\prime} j^{\prime}} .
$$

The sum over Keldysh indices $\left\{i, i^{\prime}, j, j^{\prime}\right\} \in\{r, a\}$ is easily done because $G_{a a}=0$ and a vertex function with an odd number of $a$ indices vanishes. The result is

$$
\Pi_{\mathrm{ret}}^{\mu \nu}\left(p_{0}, p\right)=i \frac{e^{2}}{2} \int \frac{d^{4} k}{(2 \pi)^{4}} \operatorname{Tr}\left[\gamma^{\mu}(\not K+m) \gamma^{\nu}(\not K-\not P+m)\right][r(K) f(K-P)+f(K) a(K-P)],
$$

where we have used the shorthand notation $r(K)$ to represent $r\left(k_{0}, k\right)$, and similarly for other propagator components. From this point on we will suppress the subscript that indicates the retarded component of the polarization tensor. In addition we consider only the relativistic limit $T \gg m$ and therefore we drop the electron mass in the integrand in Eq. (A8). We also introduce the shorthand notation $d K \equiv d^{4} k /(2 \pi)^{4}$.

We will consider a chirally asymmetric plasma which is characterized by a difference in the chemical potentials of the rightand left-handed fermions, which are denoted $\mu_{R}$ and $\mu_{L}$. We define

$$
\begin{aligned}
& \Pi_{R}^{\mu \nu}\left(p_{0}, p\right)=\left.\frac{i e^{2}}{2} \int d K \operatorname{Tr}\left[\left(1+\gamma^{5}\right) \gamma^{\mu} \not K \gamma^{\nu}(\not K-\not P)\right][r(K) f(K-P)+f(K) a(K-P)]\right|_{\mu=\mu_{R}}, \\
& \Pi_{L}^{\mu \nu}\left(p_{0}, p\right)=\left.\frac{i e^{2}}{2} \int d K \operatorname{Tr}\left[\left(1-\gamma^{5}\right) \gamma^{\mu} \not K \gamma^{\nu}(\not K-\not P)\right][r(K) f(K-P)+f(K) a(K-P)]\right|_{\mu=\mu_{L}},
\end{aligned}
$$

and

$$
\Pi^{\mu v}\left(p_{0}, p\right)=\frac{1}{2}\left[\Pi_{R}^{\mu v}\left(p_{0}, p\right)+\Pi_{L}^{\mu \nu}\left(p_{0}, p\right)\right] .
$$

The integrals in Eqs. (A9) can be rewritten by performing a shift of the 4-vector $K \rightarrow K+P$ on the term in square brackets that does not have a factor $f(K)$. All terms in the resulting 
expression have a factor $f(K)$ and, using Eq. (A3), this factor is proportional to $\delta\left(K^{2}\right)$ in the relativistic limit. The integral over $k_{0}$ can be done using this delta function. We work in timelike axial gauge and calculate only the spatial components of the polarization tensor. After doing the $k_{0}$ integral we obtain the results in Eqs. (8) and (9).

\section{APPENDIX B: NYQUIST ANALYSIS}

The dispersion equation that we have solved numerically is $\mathcal{D}=0$, where $\mathcal{D}$ is given in Eq. (28). When numerical methods are used to find the solutions of an equation, one must input the range over which the search will be performed, and solutions outside this range will be missed. A Nyquist analysis can be used to determine the number of solutions that exist so that the search range can be extended as needed. form

To explain the idea we discuss a generic equation of the

$$
f\left(p_{0}\right)=0
$$

and define the function

$$
F\left(p_{0}\right) \equiv \frac{f^{\prime}\left(p_{0}\right)}{f\left(p_{0}\right)}=\frac{d}{d p_{0}} \ln f\left(p_{0}\right) .
$$

We consider the contour integral

$$
\oint_{C} \frac{d p_{0}}{2 \pi i} F\left(p_{0}\right)
$$

where the contour is a positively (counterclockwise) oriented closed loop, which is chosen so that $F\left(p_{0}\right)$ is analytic inside the loop except at isolated points. The integral is equal to the sum of the residues. The residue of $F\left(p_{0}\right)$ at a zero of $f\left(p_{0}\right)$ of order $l$ is $l$, and the residue of $F\left(p_{0}\right)$ at a pole of $f\left(p_{0}\right)$ of order $l$ is $-l$. This gives

$$
\oint_{C} \frac{d p_{0}}{2 \pi i} F\left(p_{0}\right)=n_{Z}-n_{P}
$$

where $n_{Z}$ and $n_{P}$ are the numbers of zeros and poles of $f\left(p_{0}\right)$ inside the contour $C$, taking into account the fact that each zero and pole of order $l$ is counted $l$ times. The purpose of the Nyquist analysis is to determine $n_{Z}$.

The anisotropic dressing functions are obtained numerically from the integrals in Eqs. (18) and (19). Using $P \cdot V=$ $p\left(\hat{p}_{0}-x^{\prime}\right)$ [see Eqs. (29) and (30)] one finds that the dressing functions have a cut along the real axis for $\left|\hat{p}_{0}\right|<1$, and the dispersion equation therefore has a cut for $\left|p_{0}\right|<p$. This means that the contour $C$ can be chosen as in Fig. 11. The integrals along the lines connecting the circular contour $C_{\infty}$ to $C_{\text {cut }}$ always compensate each other and therefore the contour integral (B4) equals

$$
\oint_{C_{\infty}} \frac{d p_{0}}{2 \pi i} F\left(p_{0}\right)+\oint_{C_{\mathrm{cut}}} \frac{d p_{0}}{2 \pi i} F\left(p_{0}\right)=n_{Z}-n_{P}
$$

The contribution from the big circle is calculated by writing $p_{0}=\left|p_{0}\right| e^{i \phi}$ and taking $\left|p_{0}\right| \rightarrow \infty$ which gives

$$
\oint_{C_{\infty}} \frac{d p_{0}}{2 \pi i} F\left(p_{0}\right)=\lim _{\left|p_{0}\right| \rightarrow \infty} p_{0} F\left(p_{0}\right) \equiv n_{\infty} .
$$

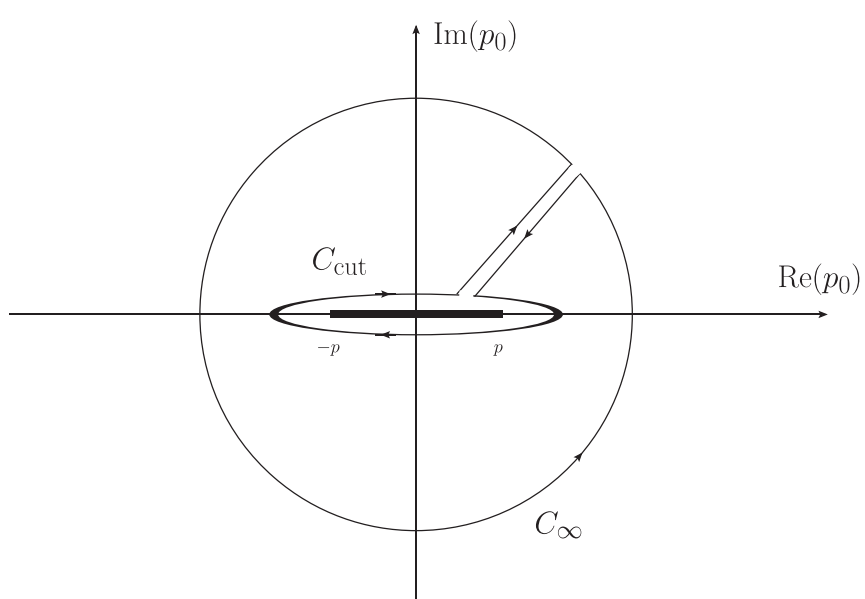

FIG. 11. The contour in the plane of complex $\hat{p}_{0}$ which is used to compute the number of solutions of the dispersion equations.

The integral along the cut can be calculated using that $F\left(p_{0}\right)$ is the logarithmic derivative of $f\left(p_{0}\right)$, which gives

$$
\begin{aligned}
\oint_{C_{\text {cut }}} \frac{d p_{0}}{2 \pi i} F\left(p_{0}\right) & =\frac{1}{2 \pi i} \oint_{C_{\text {cut }}} \frac{d}{d p_{0}} \ln f\left(p_{0}\right) \\
& =\frac{1}{2 \pi i}\left[\ln f\left(p_{0 \text { end }}\right)-\ln f\left(p_{0 \text { start }}\right)\right] \equiv n_{W},
\end{aligned}
$$

where $p_{0 \text { start }}$ is the (arbitrarily chosen) starting point of the contour which encloses the cut, and $p_{0 \text { end }}$ is the end point. The quantity $n_{W}$ is the number of times that the curve in the plane of complex $f$ travels counterclockwise around the point $f=0$, and is called a winding number. Combining the results (B6) and (B7), we have that Eq. (B5) gives

$$
n_{Z}=n_{P}+n_{\infty}+n_{W} .
$$

A Nyquist analysis of the equation $\mathcal{D}=0$ can be done analytically in the weakly anisotropic limit. When $\mu_{5}=0$ the dispersion equation in the weakly anisotropic limit factors into three separate equations

$$
\begin{aligned}
& f^{(1)}\left(p_{0}, \vec{p}\right)=P^{2}-\pi_{1}\left(p_{0}, \vec{p}\right)=0, \\
& f^{(2)}\left(p_{0}, \vec{p}\right)=\frac{1}{p_{0}^{2}}\left[p_{0}^{2}-\pi_{2}\left(p_{0}, \vec{p}\right)\right]=1-\frac{\pi_{2}\left(p_{0}, \vec{p}\right)}{p_{0}^{2}}=0, \\
& f^{(3)}\left(p_{0}, \vec{p}\right)=P^{2}-\pi_{3}\left(p_{0}, \vec{p}\right)=0 .
\end{aligned}
$$

When $\mu_{5} \neq 0$ Eqs. (B9) and (B11) are replaced by the weakly anisotropic versions of Eqs. (5) and (6), which are

$$
\begin{aligned}
f^{(4)}\left(p_{0}, \vec{p}\right)=P^{2}- & \left(\frac{1}{2}\left[\pi_{1}\left(p_{0}, \vec{p}\right)+\pi_{3}\left(p_{0}, \vec{p}\right)\right]\right. \\
& \left.+\pi_{8}\left(p_{0}, \vec{p}\right)\right)=0, \\
f^{(5)}\left(p_{0}, \vec{p}\right)=P^{2}- & \left(\frac{1}{2}\left[\pi_{1}\left(p_{0}, \vec{p}\right)+\pi_{3}\left(p_{0}, \vec{p}\right)\right]\right. \\
- & \left.\pi_{8}\left(p_{0}, \vec{p}\right)\right)=0 .
\end{aligned}
$$

In all cases it is easy to see that $n_{P}=0$. In Eq. (B10) we have divided out a zero mode, so that $n_{\infty}^{(2)}=0$, and for all of 


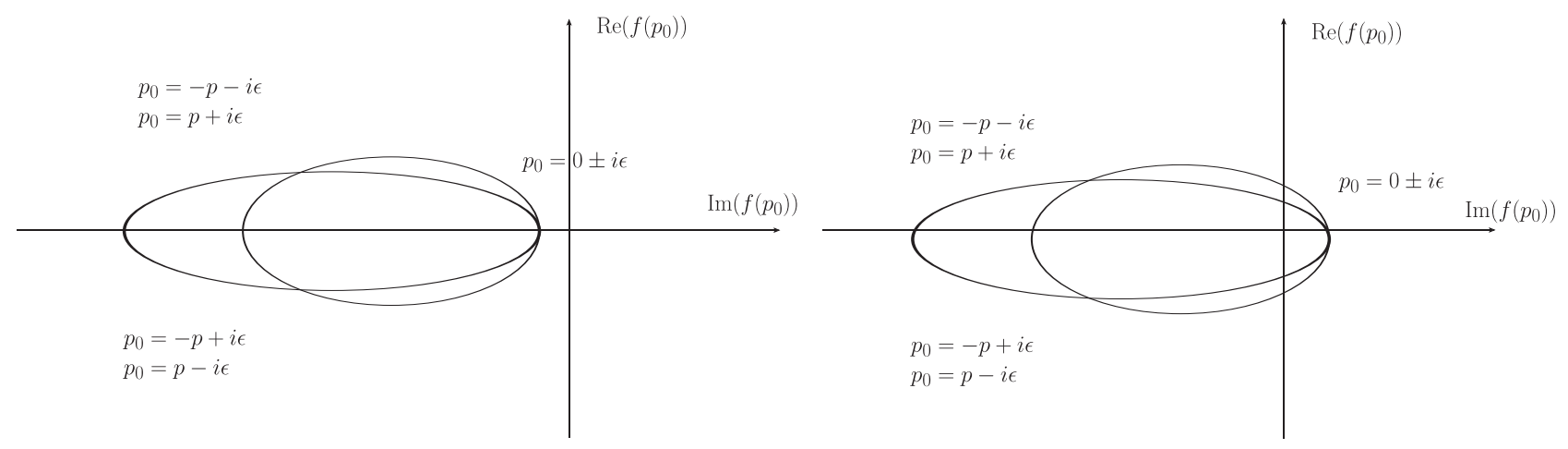

FIG. 12. Mappings of the contour $C$ in the plane of complex $p_{0}$. In the right panel the corresponding winding number is 2 , and in the left panel it is zero.

the other dispersion equations it is straightforward to see that $n_{\infty}=2$.

The nontrivial part of the calculation is the determination of the winding number, which is found by mapping the closed contour $C_{\text {cut }}$ in the plane of complex $p_{0}$ onto a path in the plane of complex $f\left(p_{0}\right)$. The points $p_{0 \text { start }}$ and $p_{0 \text { end }}$ in Eq. (B7) have the same modulus, but their phases differ by $2 \pi$. The winding number equals the number of times that the curve in the plane of complex $f$ travels counterclockwise around the point $f=0$ (some detailed examples of these mappings can be found in Ref. [9]). There are two possibilities, which are shown in Fig. 12. In the right panel, the mapping circles the origin twice, and the winding number is 2 , while in the left panel the winding number is zero. To distinguish between the two possibilities for each dispersion equation, we need to determine the sign of $\operatorname{Re}[f(0, p)]$.

We discuss first the dispersion equation (B10), which is different from the other four. In the weakly anisotropic limit we have

$$
f^{(2)}(0, \vec{p})=2+\tilde{f}\left(\xi_{i}, x, \phi\right),
$$

where $\tilde{f}\left(\xi_{i}, x, \phi\right)$ is a dimensionless function of the angular variables that depends linearly on the anisotropy parameters. Since we have assumed weak anisotropy we have $f^{(2)}(0, \vec{p})$ always positive, which means $n_{W}=2$, and there- fore Eq. (B8) gives $n_{Z}=2$. These two solutions are always present, and numerically we find they are always real. They are anisotropic modifications of the HTL longitudinal modes [denoted $\left.\pm \omega_{L}(\omega)\right]$.

For all of the remaining dispersion equations the number of solutions is

$$
n_{Z}=2+n_{W} \text {. }
$$

For Eqs. (B9) and (B11) the winding number is determined from the sign of the functions

$$
\begin{aligned}
& f^{(1)}(0, \vec{p})=-\left[p^{2}-p_{1 \text { crit }}^{2}\left(\xi_{i}, x, \phi\right)\right], \\
& f^{(3)}(0, \vec{p})=-\left[p^{2}-p_{3 \text { crit }}^{2}\left(\xi_{i}, x, \phi\right)\right] .
\end{aligned}
$$

When the chirality parameter $\mu_{5}$ is nonzero we consider Eqs. (B12) and (B13) instead of Eqs. (B9) and (B11). For weak anisotropy the winding number is determined from the sign of the functions

$$
\begin{aligned}
& f^{(4)}(0, \vec{p})=-\left[p+p_{5 \mathrm{crit}}\left(\xi_{i}, x, \phi\right)\right], \\
& f^{(5)}(0, \vec{p})=-\left[p-p_{5 \mathrm{crit}}\left(\xi_{i}, x, \phi\right)\right] .
\end{aligned}
$$

The results for $p_{1 \text { crit }}^{2}, p_{3 \text { crit }}^{2}$, and $p_{5 \text { hat }}$ are given in Eqs. (B20), (B21), and (B22):

$$
\begin{aligned}
15 p_{1 \text { crit }}^{2}= & \xi_{8} x \sqrt{1-x^{2}}\left(4 x^{2}-1\right) \cos ^{3}(\phi)+\left[\xi_{11}\left(1-2 x^{2}\right)^{2}-5 \xi_{2}\left(x^{2}-1\right)\right] \cos ^{2}(\phi)+\frac{1}{2} x \sqrt{1-x^{2}} \cos (\phi)\left[-9 \xi_{8} \cos (2 \phi)\right. \\
& \left.+10 \xi_{6}+9 \xi_{8}+6 \xi_{13}-8 \xi_{13} x^{2}\right]-\sin ^{2}(\phi)\left[5 \xi_{2}-3 \xi_{4}+\xi_{11}+9 \xi_{4}\left(x^{2}-1\right) \cos (2 \phi)+9 \xi_{4} x^{2}-3 \xi_{11} x^{2}\right] \\
& +x^{2}\left(5 \xi_{9}+6 \xi_{14}-4 \xi_{14} x^{2}\right)+2 \xi_{4}\left(-2 x^{4}+x^{2}+1\right) \cos ^{4}(\phi), \\
15 p_{3 \text { crit }}^{2}= & -3 \xi_{4} x^{2} \sin ^{2}(2 \phi)+\xi_{11}\left(2 x^{2}-1\right) \sin ^{2}(\phi)+4 \xi_{8} x \sqrt{1-x^{2}}\left(4 x^{2}-1\right) \cos ^{3}(\phi)+x \sqrt{1-x^{2}} \cos (\phi)\left[-3 \xi_{8} \cos (2 \phi)+10 \xi_{6}\right. \\
& \left.+3 \xi_{8}+4 \xi_{13}\left(3-4 x^{2}\right)\right]+5 \xi_{9}\left(2 x^{2}-1\right)+\cos ^{2}(\phi)\left[6 \xi_{4} \sin ^{2}(\phi)+5 \xi_{2}\left(1-2 x^{2}\right)+2 \xi_{11}\left(8 x^{4}-8 x^{2}+1\right)\right] \\
& +2 \xi_{4}\left(-8 x^{4}+4 x^{2}+1\right) \cos ^{4}(\phi)-2 \xi_{14}\left(8 x^{4}-12 x^{2}+3\right), \\
\frac{30}{\hat{\mu}_{5}} p_{5 \text { hat }}= & 30+10 \xi_{2}+6 \xi_{4}-5 \xi_{9}-9 \xi_{14}+10 \sqrt{1-x^{2}} x\left[3\left(\xi_{6}+\xi_{8} x^{2}\right)+\xi_{13}\left(3-4 x^{2}\right)\right] \cos (\phi) \\
& -5\left(x^{2}-1\right)\left[\xi_{4}\left(x^{2}-1\right) \cos (4 \phi)-2 \xi_{8} x \sqrt{1-x^{2}} \cos (3 \phi)+\left(3 \xi_{2}+2 \xi_{4}+\xi_{11}+4 \xi_{4} x^{2}-4 \xi_{11} x^{2}\right) \cos (2 \phi)\right] \\
& -5 x^{2}\left[3\left(\xi_{2}-2 \xi_{9}+\xi_{4} x^{2}\right)+4 \xi_{14}\left(2 x^{2}-3\right)\right]+\xi_{11}\left(20 x^{4}-15 x^{2}+2\right) .
\end{aligned}
$$



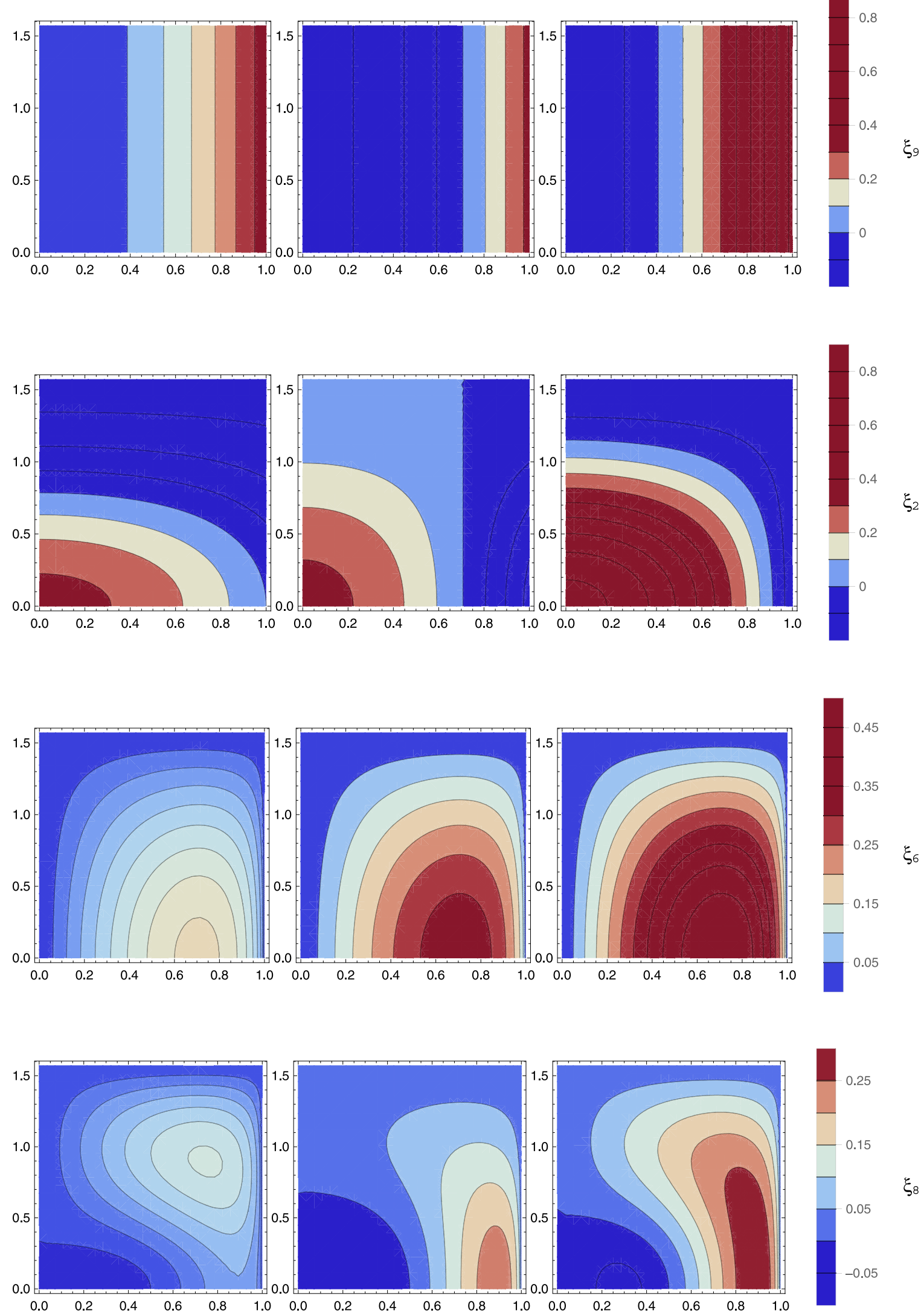

FIG. 13. Contour plots of the coefficients of the anisotropy parameters for $p_{1 \text { crit }}^{2}$ (left), $p_{3 \text { crit }}^{2}$ (center), and $p_{5 \text { crit }}$ (right). The axes show $x=\cos (\theta) \in(0,1)$ and $\phi \in(0, \pi / 2)$. 
In the isotropic case $p_{1 \text { crit }}^{2}\left(\xi_{i}, x, \phi\right)=p_{3 \text { crit }}^{2}\left(\xi_{i}, x, \phi\right)=0$, the right side of Eqs. (B16) and (B17) cannot be positive, and therefore the winding number is zero and both dispersion equations have two solutions. Remembering that in the isotropic limit $\pi_{1}=\pi_{3}=\Pi_{T}$, we see that these are the HTL transverse solutions $\left[ \pm \omega_{T}(p)\right]$. When nonzero anisotropy parameters are introduced, and when $p$ is small enough, there can be choices of the angles and anisotropy parameters for which $p_{1 \text { crit }}^{2}\left(\xi_{i}, x, \phi\right)>p^{2}>0$ and/or $p_{3 \text { crit }}^{2}\left(\xi_{i}, x, \phi\right)>$ $p^{2}>0$. In this case, the winding number is two, which means that extra solutions to Eqs. (B9) and (B11) exist.

From Eq. (B22) we have that in the isotropic limit $p_{5 \text { crit }}\left(\xi_{i}, x, \phi\right)=\hat{\mu}_{5}$, which means that $n_{W}^{(4)}=0$ and $n_{W}^{(5)}=2$ if $p<\hat{\mu}_{5}$ and zero otherwise. Thus we have that for $\mu_{5} \neq 0$ an additional pair of solutions, which turn out to be imaginary, exist even in the isotropic limit (see Fig. 1). The domain of these modes can be larger for certain orientations of the wave vector if the system is anisotropic.
The expressions in Eqs. (B20)-(B22) are only valid in the weakly anisotropic limit; however, we can use them to obtain information about the orientations of the wave vector $\vec{p}$ for which large imaginary solutions could be found with specific sets of anisotropy parameters. To obtain more insight, we can plot the coefficient of each anisotropy parameter in Eqs. (B20)-(B22). We show the results for three of the coefficients in Fig. 13. The first panel shows the dependence of the critical wave vectors on $\xi_{9}$, which is the coefficient of $\left(n_{3} \cdot v\right)^{2}$ in Eq. (16). Using our coordinate system this dot product is independent of the azimuthal angle $\phi$, and therefore we have that the terms in the critical wave vectors that are proportional to $\xi_{9}$ are also independent of $\phi$. The second panel shows the dependence of the critical wave vectors on $\xi_{2}$, which is the coefficient of $\left(n_{3} \cdot v\right)^{2}$. Both the $\xi_{9}$ and $\xi_{2}$ terms have been included in previous calculations. In the third panel we show the dependence of the critical wave vectors on $\xi_{6}$, which is the coefficient of $\left(n_{1} \cdot v\right)\left(n_{3} \cdot v\right)$, and in the last panel we show the dependence on $\xi_{8}$, the coefficient of $\left(n_{1} \cdot v\right)^{3}\left(n_{3} \cdot v\right)$.
[1] P. Romatschke and M. Strickland, Phys. Rev. D 68, 036004 (2003).

[2] P. Romatschke and M. Strickland, Phys. Rev. D 70, 116006 (2004).

[3] St. Mrówczyński, Phys. Lett. B 314, 118 (1993).

[4] P. B. Arnold, J. Lenaghan, and G. D. Moore, J. High Energy Phys. 08 (2003) 002.

[5] A. Kurkela and G. D. Moore, J. High Energy Phys. 12 (2011) 044.

[6] A. Kurkela and G. D. Moore, J. High Energy Phys. 11 (2011) 120.

[7] A. Ipp, A. Rebhan, and M. Strickland, Phys. Rev. D 84, 056003 (2011).

[8] M. Attems, A. Rebhan, and M. Strickland, Phys. Rev. D 87, 025010 (2013).

[9] M. E. Carrington, K. Deja, and St. Mrówczyński, Phys. Rev. C 90, 034913 (2014).

[10] B. Schenke and M. Strickland, Phys. Rev. D 76, 025023 (2007).

[11] B. S. Kasmaei, M. Nopoush, and M. Strickland, Phys. Rev. D 94, 125001 (2016).

[12] B. S. Kasmaei and M. Strickland, Phys. Rev. D 97, 054022 (2018).

[13] R. Baier and Y. Mehtar-Tani, Phys. Rev. C 78, 064906 (2008).

[14] P. Romatschke and M. Strickland, Phys. Rev. D 71, 125008 (2005).

[15] M. E. Carrington, K. Deja, and St. Mrówczyński, Phys. Rev. C 92, 044914 (2015).

[16] M. E. Carrington, St. Mrówczyński, and B. Schenke, Phys. Rev. C 95, 024906 (2017).

[17] A. Dumitru, Y. Guo, and M. Strickland, Phys. Lett. B 662, 37 (2008).

[18] Y. Burnier, M. Laine, and M. Vepsalainen, Phys. Lett. B 678, 86 (2009).

[19] A. Dumitru, Y. Guo, and M. Strickland, Phys. Rev. D 79, 114003 (2009).

[20] K. Boguslavski, B. S. Kasmaei, and M. Strickland, arXiv:2102.12587.
[21] M. Martinez and M. Strickland, Phys. Rev. C 78, 034917 (2008).

[22] S. Hauksson, S. Jeon, and C. Gale, Phys. Rev. C 103, 064904 (2021)

[23] B. S. Kasmaei and M. Strickland, Phys. Rev. D 102, 014037 (2020).

[24] B. S. Kasmaei and M. Strickland, Phys. Rev. D 99, 034015 (2019).

[25] Y. Akamatsu and N. Yamamoto, Phys. Rev. Lett. 111, 052002 (2013).

[26] A. Kumar, J. R. Bhatt, and P. K. Kaw, arXiv:1405.2865.

[27] K. Fukushima, D. E. Kharzeev, and H. J. Warringa, Phys. Rev. D 78, 074033 (2008).

[28] D. Kharzeev, R. D. Pisarski, and M. H. G. Tytgat, Phys. Rev. Lett. 81, 512 (1998).

[29] D. Kharzeev, A. Krasnitz, and R. Venugopalan, Phys. Lett. B 545, 298 (2002).

[30] D. Kharzeev, Phys. Lett. B 633, 260 (2006).

[31] D. E. Kharzeev, L. D. McLerran, and H. J. Warringa, Nucl. Phys. A 803, 227 (2008).

[32] D. E. Kharzeev, Ann. Phys. 325, 205 (2010).

[33] A. N. Redlich and L. C. R. Wijewardhana, Phys. Rev. Lett. 54, 970 (1985).

[34] M. Laine, J. High Energy Phys. 10 (2005) 056.

[35] J. P. Blaizot and E. Iancu, Phys. Rev. Lett. 70, 3376 (1993).

[36] P. F. Kelly, Q. Liu, C. Lucchesi, and C. Manuel, Phys. Rev. Lett. 72, 3461 (1994).

[37] D. F. Litim and C. Manuel, Phys. Rep. 364, 451 (2002).

[38] St. Mrówczyński and M. H. Thoma, Phys. Rev. D 62, 036011 (2000).

[39] St. Mrówczyński, A. Rebhan, and M. Strickland, Phys. Rev. D 70, 025004 (2004).

[40] D. T. Son and N. Yamamoto, Phys. Rev. Lett. 109, 181602 (2012).

[41] M. A. Stephanov and Y. Yin, Phys. Rev. Lett. 109, 162001 (2012).

[42] D. T. Son and N. Yamamoto, Phys. Rev. D 87, 085016 (2013). 
[43] C. Manuel and J. M. Torres-Rincon, Phys. Rev. D 89, 096002 (2014).

[44] S. Carignano, C. Manuel, and J. M. Torres-Rincon, Phys. Rev. D 98, 076005 (2018).

[45] C. Manuel and J. M. Torres-Rincon, Phys. Rev. D 92, 074018 (2015).

[46] S. Carignano and C. Manuel, Phys. Rev. D 99, 096022 (2019).

[47] S. Carignano and C. Manuel, Phys. Rev. D 103, 116002 (2021).
[48] M. Le Bellac, Thermal Field Theory (Cambridge University Press, Cambridge, 2000).

[49] R. Ghosh, B. Karmakar, and A. Mukherjee, Phys. Rev. D 102, 114002 (2020).

[50] R. L. Kobes, G. W. Semenoff, and N. Weiss, Z. Phys. C 29, 371 (1985).

[51] M. Le Bellac and H. Mabilat, Z. Phys. C 75, 137 (1997).

[52] M. E. Carrington, T. Fugleberg, D. S. Irvine, and D. Pickering, Eur. Phys. J. C 50, 711 (2007). 